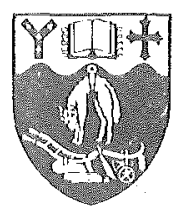

\title{
RESEARCH \\ REPORT
}

\section{NONLINEAR WAVE GROUPS IN \\ DEEP WATER}

\author{
by \\ P. J. Bryant
}

No. 13

August 1978

Department of Mathematics

University of Canterbury

Christchurch

New Zealand 
Nonlineax wave groups in deep water

By $\mathrm{P}, J$. Bryant

Nonlinear wave groups in deep water consist of wave modes for which nonlinear interactions and dispersion are in balance. The evolution equations for the wave modes are derived, and properties of nonlinear wave groups are found from these equations. It is shown that the nonlinear wave groups are linearly unstable to sideband modulations in the sense that the linearised perturbation theory, in providing a good fit over the initial time interval, predicts that the growth of the modulations is exponential. Instead the perturbed wave group is shown to return cyclically to a state close to its initial state. The cyclic recurrence is demonstrated analytically for the simpler wave groups and numerically otherwise. The interactions between nonlinear wave groups of the same and of nearly the same central wavenumbers are calculated. 


\section{Introduction}

A rave group in deep water containing a narrow waveband of wave modes propagates, to a first approximation, with the group velocity of the central wave mode in the waveband. The linear theory predicts that the wave modes will disperse slowly causing the group to disintegrate as it propagates. The nonlinear theory shows that weak nonlinear interactions between the wave modes can balance the weak dispersion so that the envelope of the wave group keeps a permanent shape.

The method of analysis used here is based on the representation of a wavetrain of periodically spaced wave groups by a Fourier series, the geries consisting of linear wave modes with amplitudes which vary slowly with time. Evolution equations for the Fourier amplitudes are calculated in terms of the nearly resonant tertiary interactions between the wave modes. This approach has its origins in the investigation by Benney and Newe11 [1] of the mechanics of the propagation of nonlinear wave envelopes. Their method was applied to deep water wave groups by Cohen, Watson and west [2], and is expanded further in the present investigation.

The alternative approach to nonlinear wave groups is by the use of model equations $[3,4,5,6]$, principally the cubic schrödinger equation. A dexivation of this equation for a narrow waveband wavetrain is to be found in [7], $\$ 17.7$, where it is shown also that such a wavetrain in deep water is unstable in the sense that small modulations to the wavetrain grow exponentially in time. Yuen and Lake [8] found good agreement between experiments on deep water narrow waveband nonlinear wave groups and the corresponding numerical solutions of the cubic Schrödinger equation, and the initial modulational instability has been demonstrated experimentally by Lake, Yuen, Rungaldier, and Ferguson [9]. The latter experiments were continued to longer times to show that the modulations are cyclic, with the wavetrain returning at regular intervals to a 
stete close to its initial state (in agreement with a previous numerical calculation by Roskes (10]). These properties are all confirmed by the present Investigation.

The inverse scattering method may be applied to the cubic Schrödinger equation $([7], \$ 17.9)$, when it is found that the nonlinear Wave groups (soliton envelopes) retain their structure after interactions with one another. This property has been verified experimentally by Yuen and Lake [8]. The method fails for nonlinear wave groups of the same central wavenumber propagating with the same velocity, so calculations are made here of the interactions between nonlinear wave groups of the same and of almost the same central wavenumbers.

\section{Evolution equations}

The water surface displacement has the form of periodically spaced wave groups, the spacing of the groups being $2 \pi \mathrm{L}$. The central wave mode of each group has wavelength $2 \pi l$, so that $k_{0}=I_{L} / l$ is the nondimensional wavenumber at the centre of the waveband, all wavenumbers being nondimensional multiples of $1 / \mathrm{L}$. The space coorainates are $x$ in the direction of wave propagation, and y vertically upwards from the mean Exee surface, both being nondimensional multiples of $l$. The time $t$ is a nondimensional multiple of $(l / g)^{\frac{1}{2}}$. The maximum trough to crest height of the wave group envelope is $2 a$, and $\varepsilon=a / l$ is the principal small parameter. The water surface displacement $\eta(x, t)$ is a nondimensional multiple of $a$, and the velocity potential $\phi(x, y, t)$ is a nondimensional multiple of $a(g l)^{\frac{1}{2}}$.

The governing equations may then be written

$$
\phi_{X X}+\phi_{Y Y}=0 \quad, y<0 \text {, }
$$




$$
\begin{aligned}
& \phi \rightarrow 0 \text { as } y \rightarrow-\infty,
\end{aligned}
$$

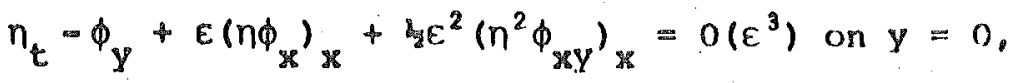

$$
\begin{aligned}
& n+\phi_{t}+\operatorname{cn} \phi_{Y t}+2 \varepsilon\left(\phi_{Y}^{2}+\phi_{Y}^{2}\right)+\operatorname{se}^{2}\left(n^{2} \phi_{Y Y}\right)_{t} \\
& +\varepsilon^{2} \eta \eta_{x t} \phi_{X}=O\left(\varepsilon^{3}\right) \text { on } y=0 \text {. }
\end{aligned}
$$

The Fourier series expansions for $n$ and $\phi$ are

$$
\begin{aligned}
& \eta=\sum_{k} g_{k}(t) \exp i\left(k x / k_{0}-w_{k} t\right)+\ldots \\
& \phi=\sum_{k} c_{k}(t) \exp \left(k y / k_{0}\right) \exp i\left(k x / k_{0}-\omega_{k} t\right)+*
\end{aligned}
$$

where denotes complex conjugate and the Fourier amplitudes $A_{k}, C_{k}$ are slow functions of $t$. The linear theory yields

$$
\begin{aligned}
& G_{k}=-i\left(k_{0} / k\right)^{\frac{k}{3}} A_{k}+o(\varepsilon) . \\
& w_{k}=\left(k / k_{0}\right)^{\frac{2}{2}} .
\end{aligned}
$$

The quadratic theory describes sum interactions between wave modes of wavenumbers $\ell, k-l$ and difference intexactions between wave modes of wavenumbers $l, k+l$ to produce wave modes of wavenumber $k$. The resulting contributions to the Fouriex amplitude $A_{k}$ are estimated by

$$
\frac{\varepsilon \omega_{k}}{\omega_{\ell}+\omega_{k-\ell}-\omega_{k}} \text { and } \frac{\varepsilon \omega_{k}}{\omega_{k+\ell}-\omega_{\ell}-\omega_{k}}
$$

respectively. None of the quadratic interactions is near resonance for the dispersion relation $(2.3 b)$, so these contributions to $A_{k}$ are only $O(\varepsilon)$ compared with $A_{k}$ itself.

The tertiary interactions may be separated into two kinds, those that are significant because they are near resonance, and those whose contribution to $A_{k}$ is not significant because they are not near resonance. When the three interacting wave modes as well as the resulting wave mode all have wavenumbers in the neighbourhood of $\mathrm{k}_{0}$, their contribution to $A_{\mathrm{k}}$ is estimated by (equation 2.6 ) 


$$
\frac{\varepsilon^{2} w_{k}}{u_{m}+w_{k+l-m}-w_{l}-w_{k}} .
$$

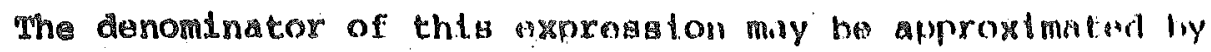

$$
\left(\frac{d^{2} \omega}{d k^{2}}\right)_{k_{0}}\left(\left(m-k_{0}\right)^{2}+\left(k+l-m-k_{0}\right)^{2}-\left(l-k_{0}\right)^{2}-\left(k-k_{0}\right)^{2}\right) .
$$

The above contribution to $A_{k}$ is $0(1)$, of the same magnitucle as $A_{k} i$ tseli, when all wavenumbers $k$ in the narrow waveband centred on $k_{0}$ are such that

$$
\frac{\left(k-k_{0}\right)^{2}}{k_{0}^{2}} \sim \varepsilon^{2}
$$

The amplitude paramter $\varepsilon$ is muen smaller than 1 , so for this relation to be satigfied, $k_{0}$ must be much greater than 1 . For this reason, the present investigation is restricted to narrow wavebands centred on wavenumbers $k_{0} \Rightarrow 1$ for which significant tertiary interactions occur between the wave modes present.

None of the remaining tertiary interactions lies near resonance, so none contributes significantly to $A_{k}$. One such tertiary interaction is the sum interaction between three of the wave modes present to produce an $O\left(\varepsilon^{2}\right)$ wave mode with a wavenumber near $3 k_{0}$. A less obvious tertiary interaction is that between a quadraticaliy generated $O(\varepsilon)$ wave mode and an $O(1)$ linear wave mode to produce an $O\left(\varepsilon^{2}\right)$ wave mode, as for example when the $O(\varepsilon)$ correction to equation (2.3a) is multiplied with an $O(1)$ amplitude $A$ in the expansion of the thixd term of equation (2.1c). The derivation of the evolution equations is now straightforward. The Fourier series $(2,2 a, b)$ are substituted into equations $(2.1 c, d)$, the quadratic terms are neglected completely, and the only tertiaxy terms retained are those for quartets of wavenumbers $k, l, m, k+l-m$ in the neighbourhood of $\mathrm{k}_{0}$, with the Fourier amplitudes $\mathrm{C}$, $\mathrm{A}$ in the tertiary terms related by equation (2.3a). Equations (2.1c,d) become (with $\mathrm{p}=\mathrm{k}+\ell-\mathrm{m})$ 


$$
\begin{aligned}
& A_{k}^{\prime}-i u_{k} A_{k}-k c_{k}+\frac{i k \varepsilon^{2}}{8 k_{0}^{2}} \sum_{\ell=1}^{\infty} \sum_{m=1}^{k+l-1}\left(l \omega_{\ell}+m u_{m}+p \omega_{p}\right) A_{\ell}^{k} A_{m} A_{p} x \\
& \exp -i\left(\omega_{\mathrm{m}}+\omega_{p}-()_{\ell}-\left(w_{k}\right) t=0,\right. \\
& A_{k}+c_{k}^{\prime}-1 \omega_{k} c_{k}+\frac{\varepsilon^{2}}{8 k_{0}^{2}} \sum_{\ell=1}^{\infty} \sum_{m=1}^{k+\ell-1}\left\{\left(\omega_{\ell}-\omega_{m}-\omega_{p}\right)\left(-\ell \omega_{\ell}+m \omega_{m}+p \omega_{p}\right)\right. \\
& \left.+2\left(m \omega_{m} \omega_{p}+l \omega_{\ell} \omega+p \omega_{\ell} \omega_{p}\right)\right\} A_{\ell}^{*} A_{p} \exp -i\left(\omega_{m}+\omega_{p}-\omega_{l}-\omega_{k}\right) t=0 .
\end{aligned}
$$

The amplitude $C_{k}$ is eliminated now between these two equations, and the symmetry between in and $k+l-m(=p)$ used to simplify the interactior coefficlent, when the evolution equation for $A_{k}$ is found to be

$$
\begin{array}{r}
A_{k}^{\prime}=1 \varepsilon^{2} \sum_{l=1}^{\infty} \sum_{m=1}^{k+l-1} R_{k l m} A_{l}^{\prime} A_{p} \exp -i\left(\omega_{m}+w_{p}-w_{l}-w_{k}\right) t_{0} \\
k_{0}-k_{1} \leqslant k \leqslant k_{0}+k_{1} .
\end{array}
$$

where

$$
R_{k \ell m}=\frac{-k\left\{(k+l) \omega_{m} \omega_{p}+(3 \ell+m) \omega_{l} \omega_{m}+(3 \ell+p) \omega_{\ell} \omega_{p}-2 \ell \omega_{l}^{2}\right\}}{8 k_{0}^{2}\left(\omega_{m}+\omega_{p}-\omega_{l}+\omega_{l k}\right)} .
$$

Each $w$ in this formula is given by equation $(2.3 \mathrm{~b})$. Since the wavenumbers $m, p, l, k$ all lie in a narrow waveband near $k_{0}$, the interaction coefficient $R_{k \ell m}$ may be approximated by

$$
R_{k \ell m}=-\frac{1}{2}\left(1+\frac{9\left(k-k_{0}\right)}{8 k_{0}}+\frac{11\left(l-k_{0}\right)}{8 k_{0}}\right)+0\left(\frac{k-k_{0}}{k_{0}}\right)^{2}
$$

An envelope function $F(x, t)$ is introduced by rewriting the Fourier series $(2.2 a)$ as

$$
\eta \quad=\frac{1}{2} F(x, t) \exp i(x-t)+*, \quad\left(\omega_{k_{0}}=1\right)
$$

where

$$
F(x, t)=\sum_{k_{0}-k_{1}}^{k_{0}+k_{1}} A_{k}(t) \exp i\left(\frac{k-k_{0}}{k_{0}} x-\left(w_{k}-1\right) t\right)
$$


If the interaction coeffictent $\mathbb{K}_{K \ell_{\mathrm{m}}}$ is approximatad by $-\frac{1}{2}$ and the d1 aperion relation $(2.3 b)$ is approxlmated by

$$
\begin{aligned}
w_{k} & =w_{k_{0}}+\left(k-k_{0}\right)\left(\frac{d \omega}{\partial k}\right)_{k_{0}}+2\left(k-k_{0}\right)^{2}\left(\frac{d^{2} \omega}{d k^{2}}\right)_{k_{0}} . \\
& =1+\frac{k-k_{0}}{2 k_{0}}-\frac{\left(k-k_{0}\right)^{2}}{8 k_{0}^{2}} .
\end{aligned}
$$

it is found from equation $(2.6 \mathrm{a})$ that $F(x, t)$ satisfies the cubic Schröđinger equation

$$
\frac{\partial F}{\partial t}+\frac{1}{2} \frac{\partial F}{\partial x}+\frac{i}{8} \frac{\partial^{2} F}{\partial x^{2}}+\frac{1}{2} i \varepsilon^{2} F^{2} F^{2}=0 .
$$

The first two terms of this equation describe propagation of $F$ with the group velocity $\frac{1}{2}$, and the last two terms, which from relation (2.4) are of comparable small magnitude, describe the weak dispersion and the weak nonlinearity respectively. Most previous investigations have discussed nonlinear wave groups as solutions of the cubic sehrödinger equation (2.9), but the present investigation concentrates on those properties of nonlinear wave groups which are obtained from the evolution equations $(2.6)$.

\section{Wave groups}

The wave group solutions of the evolution equations are now calculated. The two parameters describing the shape of a wave group are the nondimensional amplitude $\varepsilon$ and the nondimensional central wave number $k_{0}$. Relation (2.4) for the width of the waveband indicates that the number of wavelengths in each group is proportional to $1 / \varepsilon$, and hence that the ratio of the length of the group to the distance between groups is proportional to $1 /\left(\mathrm{k}_{0} \varepsilon\right)$. The propagation velocity of the group tends towards the group velocity at wavenumber $k_{0}$ in the linit 
a $\varepsilon$ tends to zero, this velocity being $\frac{1}{2}$ in the nondimensional multiples uged. The simplest method of ensuring that in the wave group calculations the centre of the waveband remains in the neighbourhood of $k_{0}$ is by setting the propagation velocity equal to $\frac{1}{2}$ for all values of $\varepsilon$. The peak of the wavenumber spectrum then changes continuously in position as $\varepsilon$ is increased, but remains in the neighbourhood of $k_{1}$.

The Fourier series describing nonlinear wave groups propagating with velocity $\frac{1}{2}$, containing waves of central wavenumber 1 and central frequency 1, is

$$
n(x, t)=\frac{1}{2}\left(\sum_{k_{0}-k_{1}}^{k_{0}+k_{1}} a_{k} \exp i \frac{k-k_{0}}{k_{0}}\left(x-\frac{1}{2} t\right)\right) \exp i(x-t) \exp -i \alpha \varepsilon^{2} t+k_{0}
$$

where from equation (2.2a),

$$
A_{k}(t)=a_{k} \exp i\left(\omega_{k}-1-\frac{k-k_{0}}{2 k_{0}}-\alpha \varepsilon^{2}\right) t, \quad k_{0}-k_{1} \leqslant k \leqslant k_{0}+k_{1} .
$$

The amplitudes $a_{k}$ are constants, the first three terms of the phase of $A_{k}$ are together $0\left(\varepsilon^{2} t\right)$ (from equations $(2.4)$ and $(2.8)$ ), and the unknown part of the phase is written $\alpha E^{2} t$ to give $A_{k}$ the slow time dependence indicated by the evolution equations $(2.6)$.

The equations determining $a_{k}\left(k_{0}-k_{1} \leqslant k \leqslant k_{0}+k_{1}\right)$ and $\alpha$ are therefore

$$
\begin{array}{r}
\left(\left(w_{k}-1-\frac{k-k_{0}}{2 k_{0}}-\alpha \varepsilon^{2}\right) / \varepsilon^{2}\right) a_{k}=\sum_{l} \sum_{m} R_{k l m} a_{l} a_{m} a_{k+l-m} \\
k_{0}-k_{1} \leqslant k \leqslant k_{0}+k_{1} .
\end{array}
$$

together with the geometric constraint imposed by the definition of $\varepsilon$, namely

$$
\sum_{k_{0}-k_{1}}^{k_{0}+k_{1}} a_{k}=1
$$

For any given $k_{0}$ and $\varepsilon$, thes $2 k_{1}+2$ equations are solved for the $2 k_{1}+2$ variables $a_{k}\left(k_{0}-k_{1} \leqslant k \leqslant k_{0}+k_{1}\right)$ and $\alpha$ by a generalised 
Newton-Raphson technique. The bandwidth $2 k_{1}$ is increased step by step until no further change occurs in all variables to the precision required (usual1y $10^{-4}$ ).

The nonlinear wave group for $k_{0}=40, \varepsilon=1 / 40$, is sketched in figure 1. The amplitudes $a_{k}$ are plotted in figure $I(a)$, and $\alpha=0.251$. One wavelength of the corresponding water surface displacement $\eta$ is sketched in figure $1(b)$. The amplitudes $a_{k}$ and water surface displacement $n$ for $k_{0}=40, \varepsilon=1 / 10$ are sketched in figure 2 for comparison. and $\alpha=0.262$ in this example. The solitary nonlinear wave group solutions of the cubic Schrödinger equation (2.9) may be written

$$
n(x, t)=\operatorname{sech} \sqrt{2} \varepsilon\left(x-\frac{1}{2} t\right) \cos \left(x-t-\frac{1}{4} \varepsilon^{2} t\right) \text {. }
$$

for which

$$
a_{k}=\frac{1}{2 \sqrt{2} k_{0} \varepsilon} \operatorname{sech} \frac{\pi\left(k-k_{0}\right)}{2 \sqrt{2} k_{0} \varepsilon}
$$

and

$$
\alpha=\frac{1}{4}
$$

There is good agreement between the solutions in figures 1 and 2 , and equations (3.3), apart from a slight asymmetry present in the sketched solutions for $a_{k}$. It is noted in this respect that the leading terms in the omitted part of the contributions of the interaction coefficient (2.7) and the Taylor series $(2.8)$ to the cubic Schrödinger equation are both skew symmetric in $k-k_{0}$. 


\section{Linear instahl11ty}

Benjamin and Feir [11] showed that a progressive wavetrain in deep water is unstable, in the sense that a sideband modulation grows exponentialy in time during the initial time interval in which the amplitude of the modulation remains sma11. Lake et al [9] found more recently that over longer times the amplitude of the modulation increases and decreases, with the modulated wavetrain returning cyclically close to its initial state.

Benjamin and Feir analysed a wavetrain of fundamental wavenumber $k$ and frequency $w$ interacting with sideband wave modes of wavenumbers $k(1 \pm k)$ and Irequencies $w(1 \pm \delta)$ where $k$ and $\delta$ are small fractions. The essence of the instability mechanism is that a significant interaction occurs within the quartet with wavenumbers $k, k, k(1+k), k(1-k)$ and frequencies $\omega, \omega, \omega(1+\delta), \omega(1-\delta)$ respectively, causing the two sideband wave modes to grow at the expense of the fundamental wave mode. In a nonlinear wave group, significant interactions occur within all quartets with wavenumbers $k, l, m, k+l=m$ in the neighbourhood of $k_{0}$. If one such wave mode is perturbed or is itself a small anglitude modulation, it can be expected therefore that the significant interaction with the other three wave modes will cause this perturbation or modulation to grow exponentially. This property is now confirmed. It can be expected also that over longer times the perturbed nonlinear wave group will return cyclically close to its initial state, and this property is confirmed in the following two sections.

The Fourier series for a given nonlinear wave group is that stated in equation (3.1a). In order to investigate the stability and evolution of a perturbed nonlinear wave group, it is preferable to use a Fourier sexies for the water surface displacement which has the same form as equation (3.1a): Equation (2.2a) is therefore replaced by 


$$
\eta=\frac{1}{2} \sum_{k} B_{k}(t) \exp i \frac{k-k_{0}}{k_{0}}\left(x-\frac{1}{2} t\right) \operatorname{exg} i(x-t) \exp -i \alpha \varepsilon^{2} t+w_{0}
$$

where

$$
A_{k}(t)=B_{k}(t) \exp i\left(u_{k}-1-\frac{k-k_{0}}{2 k_{0}}-\alpha \varepsilon^{2}\right) t \text {. }
$$

The evolution equation for $\mathrm{B}_{\mathrm{k}}$, from equation $(2.6 \mathrm{a})$, is

$$
B_{k}^{*}-i \varepsilon^{2} B_{k} B_{k}=i \varepsilon^{2} \sum_{l} \sum_{m} R_{k l m} B_{l}^{*} B_{m} B_{k+l-m}
$$

where

$$
\begin{aligned}
p_{k} & =-\left(\left(k_{k}-1-\frac{k-k_{0}}{2 k_{0}}\right) / \varepsilon^{2}+\alpha\right. \\
& =\frac{1}{8}\left(\frac{k-k_{0}}{k_{0} \varepsilon}\right)^{2}+\alpha \text { approximately, for wavenumbers } k \text { near } k_{0}
\end{aligned}
$$

The wavenumber of the perturbing wave mode is denoted by $k_{0}$ where $k_{0}-k_{1} \leqslant K \leqslant k_{0}+k_{1}$, and $i t s$ comples amplitude is $a_{K}+\hat{\theta}_{K}$ if $K$ coincides with one of the (integer) wavenumbers present, or is $\hat{B}_{K}$ otherwise. Linearisation with respect to $\hat{B}$ in the tertiary interaction terms of equation (4.2) indicates that the perturbed wave modes present have wavenumbers $k+n, n=0, \pm 1, \pm 2, \ldots$, and $2 k_{0}-k+n, n=0, \pm 1, \pm 2, \ldots$ The evolution equations, linearised with respect to the perturbations $\hat{B}$, are

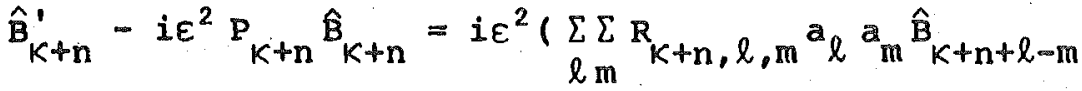

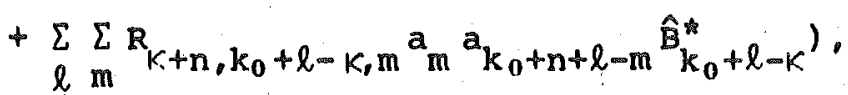

$$
\begin{aligned}
& \hat{B}_{2 k_{0}-k+n}^{\prime}-i \varepsilon^{2} P_{2 k_{0}-k+n} \hat{B}_{2 k_{0}-k+n}=i \varepsilon^{2}\left(\sum_{\ell} \sum_{m} R_{2 k_{0}-k+n, l, m}{ }^{a} l_{m}^{a} \hat{B}_{2 k_{0}-k+n+l-m}\right. \\
& \left.+\sum_{l m} \sum_{2 k_{0}-k+n, k+l-k_{0}, m} a_{m} a_{k_{0}+n+l-m} \hat{B}_{K+l-k_{0}}^{k}\right) .
\end{aligned}
$$

The system of first order linear differential equations with constant coefficients consisting of equations $(4.4 a)$ with the complex conjugates of equations $(4.4 \mathrm{~b})$ is a complete set of equations for the complex 
amplitudes $\hat{\mathrm{B}}_{K+n^{\prime}} \mathrm{n}=0, \pm 1, \pm 2, \ldots$, and $\hat{\mathrm{B}}_{2 k_{0}-K+n^{\prime}} n=0, \pm 1, \pm 2, \ldots$

The method of solution of this system of differential equations for any given values of $\varepsilon, k_{0}$ and $k$ has been described previously ( [12], \$4), and is as follows. The systen of differential equations is reduced to a system of algebraic equations by seeking normal mode solutions for which each amplitude $\hat{B}$ has the same slow time dependence $\exp \left(i \lambda \varepsilon^{2} t\right)$. The system is then solved for the range of wavenumbers within the waveband $k_{0}-k_{1} \leqslant k \leqslant k_{0}+k_{1}$. The width $2 k_{1}$ of the waveband is increased step by step until the common eigenvalues $\lambda$ of consecutive sets of equations agree to within an arbitrary numerical precision. The system is stable if all eigenvalues $\lambda$ are real, unstable if any paix of eigenvalues are complex conjugates. The solutions for the amplitudes $\hat{B}$ resulting from any givan initial conditions may be calculated from the eigenvectors of the set of algebxaic equations.

Examples have been calculated for a range of values of $\varepsilon_{0} k_{0}$, and $k_{i}$ and in every example there was at least one pair of eigenvalues $\lambda$ that were comples conjugates. The conclusion is that nonlinear wave groups are unstable to small pertubations of the wave modes composing them, and are unstable to small amplitude sideband modulations at other wavenumbers near $k_{0}$. The amplitudes of the unstable modes were found to decrease as $K$ moved away from $k_{0}$, which is to be expected because the interactions within the quartets of wavenumbers then move further from resonance.

The eigenvector corresponding to each eigenvalue $\lambda$ is dominated for almost every $\lambda$ by a unique complex amplitude $\hat{B}_{k}$, enabling $\lambda$ to be identified with a unique wavenumber $k$. This identification leads to an intexpretation for $\lambda$ in terms of a coefficient $q_{k}$ for those wavenumbers $k$ outside the immediate neighbourhood of $k_{0}$. When all terms proportional to $\hat{B}_{K+n}$ in equation $(4.4 a)$ are included in the left hand 
side of this equation, this side may be rewritten $\hat{B}_{K+n}^{\prime}-i \varepsilon^{2} \bigcap_{k+n} \hat{B}_{K+n}$ where

$$
\Omega_{\mathrm{k}}=\mathrm{P}_{\mathrm{k}}+2 \sum_{\ell} \mathrm{R}_{\mathrm{k} \ell \ell} \mathrm{a}_{\ell}^{2}
$$

The normal mode solutions of equation $(4.4 a)$ are those solutions for which $\hat{B}_{k}^{0}=i \varepsilon^{2} \lambda \hat{B}_{k}$. It was found in all the examples calculated that $\lambda$ tended towards the corresponding value of $q_{k}$ as $k$ tended away from $k_{0}$. The values of $\lambda$ and $\Omega_{k}$ are listed in the following table for the first example described below, for which $k_{0}=k=100$.

$\begin{array}{cccccccccccc}k & 95 & 96 & 97 & 98 & 99 & 100 & 101 & 102 & 103 & 104 & 105 \\ \mathrm{O}_{k} & 3.243 & 2.076 & 1.174 & 0.535 & 0.153 & 0.025 & 0.146 & 0.515 & 1.125 & 1.975 & 3.061 \\ \lambda & 3.245 & 2.079 & 1.180 & 0.550 & & 0.250 & & 0.530 & 1.131 & 1.978 & 3.063\end{array}$

Table 1 .

The complex pair of eigenvalues $(1.25 \pm 2.63 i) \times 10^{-4}$ has a pair of complex eigenvectors which contribute significantly to all three central amplitudes $\hat{B}_{99}, \hat{B}_{100}, \hat{B}_{101} . A s k$ tends away from $k_{0}$ and $\lambda$ tends towards $o_{k}$, the contribution of the normal mode solution to $n_{\text {, from equation }}$ (4.1a), is dominated by

$$
\frac{1}{2} \hat{b}_{k} \exp i\left\{k x-\left(\omega_{k}+\varepsilon^{2} \sum_{l} a_{\ell}^{2}\right) t\right\}+*
$$

where $\hat{b}_{k}$ is a constant, and $R_{k l l}$ is approximated by $-\frac{1}{2}$ (equation 2.7). It follows that perturbations or modulations with wavenumbers outside the immediate neighbourhood of $k_{0}$ propagate almost as linear waves, with only an $O\left(\varepsilon^{2}\right)$ correction to their linear frequency of similar form to the amplitude correction for the frequency of stokes waves in deep water. This is consistent with the property that nonlinear interactions are significant only in the immediate neighbourhood of $k_{0}$ (relation 2.4). The first example to be described is that for which $\varepsilon=0.01$, $k_{0}=k=100$. The envelope of the wave group is almost identical 
with that in figure 1 , since $k_{0} E=1$ in both cases. When the pexturbation is applied to one of the existing wave modes, the set of wavenumbers of the perturbations coincides with the set of wavenumbers of the group itself. The perturbation variables are $\hat{\mathrm{B}}_{\mathrm{k}}, \hat{\mathrm{B}}_{\mathrm{k}}^{*}$, $\mathrm{k}_{0}-\mathrm{k}_{1} \leqslant \mathrm{k} \leqslant \mathrm{k}_{0}+\mathrm{k}_{1}$, which means that the real eigenvalues occur in pairs, $\pm \lambda$, and the complex eigenvalues occur as $\pm\left(\lambda_{x} \pm i \lambda_{i}\right)$. If the initial conditions are $\hat{B}_{100}=\hat{b}_{,} \hat{B}_{k}=0$ otherwise, the solution for the three central perturbations (with the eigenvalues listed in and below table 1) is found to be

$$
\begin{aligned}
\hat{\text {Bg99}}= & \hat{b}\left[(-197.45+212.24 i) \exp i\left(\lambda_{r}+i \lambda_{i}\right) \varepsilon^{2} t\right. \\
& +(-197.45-212.24 i) \exp i\left(\lambda_{r}-i \lambda_{i}\right) \varepsilon^{2} t \\
& +(197.77+212.90 i) \exp i\left(-\lambda_{r}+i \lambda_{i}\right) \varepsilon^{2} t \\
& +(197.77-212.90 i) \exp i\left(-\lambda_{r}-i \lambda_{i}\right) \varepsilon^{2} t \\
& -0.37 \exp i \lambda_{100} \varepsilon^{2} t-0.33 \exp -i \lambda_{1000} \varepsilon^{2} t \\
& +0.08 \exp i \lambda_{98} \varepsilon^{2} t-0.02 \exp -i \lambda_{102} \varepsilon^{2} t+\ldots(0.006) 1,
\end{aligned}
$$

$$
\begin{aligned}
\hat{B}_{100}= & \hat{b}\left[(16.85+547.58 i) \exp i\left(\lambda_{r}+i \lambda_{i}\right) \varepsilon^{2} t\right. \\
& +(16.85-547.58 i) \exp i\left(\lambda_{r}-i \lambda_{i}\right) \varepsilon^{2} t \\
& +(-16.85+547.61 i) \exp i\left(-\lambda_{r}+i \lambda_{i}\right) \varepsilon^{2} t \\
& +(-16.85-547.61 i) \exp i\left(-\lambda_{r}-i \lambda_{i}\right) \varepsilon^{2} t \\
& +1.40 \exp i \lambda_{100} \varepsilon^{2} t-0.41 \exp -i \lambda_{100} \varepsilon^{2} t \\
& +0.01 \exp i \lambda_{98} \varepsilon^{2} t-0.01 \exp -i \lambda_{98} \varepsilon^{2} t \\
& +0.01 \exp i \lambda_{i 02} \varepsilon^{2} t-0.01 \exp -i \lambda_{102} \varepsilon^{2} t+\ldots(0.0005) 1,
\end{aligned}
$$

$$
\begin{aligned}
\hat{B} 101= & \hat{b} \\
& +(223.25+441.07 i) \exp i\left(\lambda_{r}+i \lambda_{i}\right) \varepsilon^{2} t \\
& +(-222.96+440.75 i) \exp i\left(-\lambda_{r}+i \lambda_{i}\right) \varepsilon^{2} t \\
& +(-222.96-440.75 i) \exp i\left(-\lambda_{r}-i \lambda_{i}\right) \varepsilon^{2} t \\
& -0.30 \exp i \lambda_{100} \varepsilon^{2} t-0.34 \exp -i \lambda_{100} \varepsilon^{2} t \\
& \left.+0.08 \exp i \lambda_{102} \varepsilon^{2} t-0.02 \exp -i \lambda_{98} \varepsilon^{2} t+\ldots(0.006)\right]: \quad \text { (4.7c) }
\end{aligned}
$$


(The figure in brackets at the end of each equation is the magnitude of the next term in the expansion.)

The complete complex amplitudes are $B_{k}=a_{k}+\hat{B}_{k}$, and the three values of $a_{k}$ to be added to the above equations axe $a_{99}=0.207$. $a_{100}=0.353, a_{101}=0.213$. The moduli and arguments of $B_{99}, B_{100}$ $B_{101}$, for the initial perturbation amplitude $\hat{b}=0.01$, are plotted in figure 3. The dashed curves are the complex amplitudes predicted by the linearised model, including the perturbations given by equations (4.7). The solid curves are the complex amplitudes without linearisation, obtained by a numerical solution of the evolution equations (4.2) from the same initial conditions as the linearised solution.

The curves for the moduli are of most interest because they have a direct influence on the shape of the group envelope. They demonstrate that the linearised theory fits well over the initial interval of slow time, but in so doing predicts incorrectly that the perturbations will continue to grow exponentially. The full solution without linearisation shows that the moduli of these amplitudes return to a state close to their initial state. These curves illustrate the important property that the prediction of instability by the linearised model over short times is not applicable to the development of the wave system over longer times. A better fit between the linearised model and the full solution is found for the arguments of the complex amplitudes. The nearly constant negative rate of change of these arguments corresponds to an increase in the phase velocity of each of the calculated wave modes.

The second example described is that for which $\varepsilon=0.01, k_{0}=100$, $K=99.8$. This example has been chosen because it shows the structure of the perturbation solution when the sideband modulation is applied at a wavenumber which is not already present. The perturbation variables are $\hat{\mathrm{B}}_{\mathrm{k}-0.2}, \mathrm{k}_{0}-\mathrm{k}_{1}<\mathrm{k} \leqslant \mathrm{k}_{0}+\mathrm{k}_{1}$, and $\hat{\mathrm{B}}_{\mathrm{k}+0.2}^{\mathrm{t}}, \mathrm{k}_{0}-\mathrm{k}_{1} \leqslant \mathrm{k}^{2}<\mathrm{k}_{0}+\mathrm{k}_{1}$. The 
eigenvalues $\lambda$ tend towards the corresponding values of $\Omega_{k \pm 0.2}$ as $k$ tends away from $k_{0}$, as in the first example. The central eigenvalues are $\lambda_{98.2}=-0.457, \lambda_{99.8}=0.268, \lambda_{100.2}=-0.263, \lambda_{101.8}=0.441$, with the complex set $-6 \times 10^{-4} \pm 0.016 i, 3 \times 10^{-4} \pm 0.012 i$ favouring all six central wavenumbers $99 \pm 0.2,100 \pm 0.2,101 \pm 0.2$. The two central modulations starting from initial conditions $\hat{B}_{99.8}=\hat{B}_{100.2}=\hat{b}$, $\hat{\mathrm{B}}_{\mathrm{k} \pm 0.2}=0$ otherwise, are

$$
\begin{aligned}
& \hat{B}_{99.8}=6\left[(-1.367+8.869 i) \exp i\left(-6 \times 10^{-4}+0.016 i\right) \varepsilon^{2} t\right. \\
& +(-1.367-8.869 i) \exp i\left(-6 \times 10^{-4}-0.016 i\right) \varepsilon^{2} t \\
& +(1.447+10.026 i) \exp i\left(3 \times 10^{-4}+0.012 i\right) \varepsilon^{2} t \\
& +(1.447-10.026 i) \exp i\left(3 \times 10^{-4}-0.012 i\right) \varepsilon^{2} t
\end{aligned}
$$

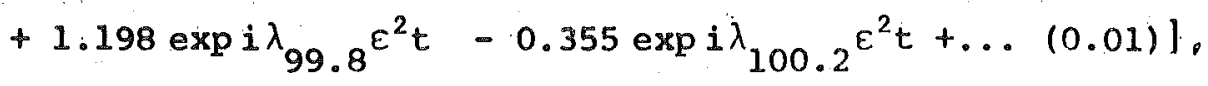

$$
\begin{aligned}
& \hat{B}_{100.2}^{n}=6\left[(-1.244-8.962 i) \exp i\left(-6 \times 10^{-4}+0.016 i\right) \varepsilon^{2} t\right. \\
& +(-1.244+8.962 i) \exp i\left(-6 \times 10^{-4}-0.016 i\right) \varepsilon^{2} t \\
& +(1.284-10.084 i) \exp i\left(3 \times 10^{-4}+0.012 i\right) \varepsilon^{2} t \\
& +(1.284+10.084 i) \exp i\left(3 \times 10^{-4}-0.012 i\right) \epsilon^{2} t
\end{aligned}
$$

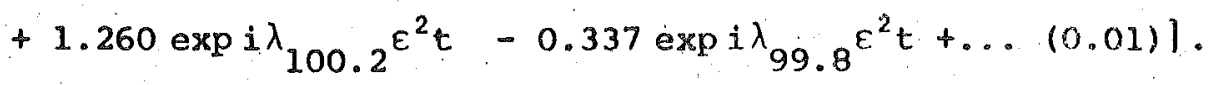

The linearised solution could not be compared with a full solution of the evolution equations because the large number of fractional wavenumbers placed the calculation beyond the computing facilities available. However, since the nearly resonant mechanism of energy transfor withill quartets which applied in the previous example still applies here, it can be expected that the above solution is valid only for an initial interval in slow time, and that cyclic recurrence will occur over longer times. 


\section{An analytical solution of the evolution equations}

A nonlinear wave group of small amplitude, such as that in the examples of the previous section, is dominated by the interactions between its three central wave modes. The evolution equations (4.2) are solved therefore for a nonlinear wave system consisting only of the three central wave modes.

The coefficients in equations (4.2) are approximated and rewritten as follows,

$$
\begin{aligned}
& P_{k_{0}}=\frac{1}{4}=P_{0} \\
& P_{k_{0} \pm 1}=\frac{1}{8\left(k_{0} \varepsilon\right)^{2}}+\frac{1}{4}=P_{1} \quad \text { (from equation 4.3b). } \\
& \left.R_{k_{l m}}=-\frac{1}{2} \quad \text { (from equation } 2.7\right) .
\end{aligned}
$$

The time variable is changed to $\tau=\varepsilon^{2} t$. The three complex amplitudes are rewritten

$$
\mathrm{B}_{\mathrm{k}_{0}-1}=\mathrm{X}_{0} \quad \mathrm{~B}_{\mathrm{k}_{0}}=\mathrm{Y}_{0} \quad \mathrm{~B}_{\mathrm{k}_{0}+1}=\mathrm{Z} .
$$

The evolution equations (4.2) then become

$$
\begin{aligned}
& X^{\prime}-i P_{1} X=-\frac{1}{2} i\left\{\left(|X|^{2}+2|Y|^{2}+2|z|^{2}\right) X+Y^{2} Z^{*}\right\} \\
& Y^{\prime}-i P_{0} Y=-\frac{1}{2} i\left\{\left(2|X|^{2}+|Y|^{2}+2|z|^{2}\right) Y+2 X Y^{*} Z\right\} \\
& z^{\prime}-i P_{1} Z=-\frac{1}{2} i\left\{\left(2|X|^{2}+2|Y|^{2}+|Z|^{2}\right) Z+Y^{2} X^{*}\right\}
\end{aligned}
$$

The steady solution of these equations is the nonlinear wave group whose amplitudes are given by

$$
\begin{aligned}
x^{2}=z^{2} & =\frac{1}{60}\left(4-\frac{1}{\left(k_{0} \varepsilon\right)^{2}}\right)=a_{1}^{2} . \\
y^{2} & =\frac{1}{10}\left(1+\frac{1}{\left(k_{0} \varepsilon\right)^{2}}\right)=a_{0}{ }^{2} .
\end{aligned}
$$

Two first integrals of equations (5.1) may be found immediately.

These are

$$
\begin{aligned}
|x|^{2}+|y|^{2}+|z|^{2} & =C_{1}, \\
|x|^{2}-|z|^{2} & =C_{2},
\end{aligned}
$$


where $C_{\mathbb{l}}, C_{2}$ are constants, When the combination $Y^{\prime \prime} Y^{*}-Y^{*} Y^{\prime}$ is formed and the right hand side simplified using equation (5.3a), it may be integrated to yield

$$
Y^{\prime} Y^{*}-y^{\prime \prime} Y=\frac{1}{4} i|Y|^{4}+i\left(\frac{1}{2}+\frac{1}{4\left(k_{0} \varepsilon\right)^{2}}-\frac{3 C_{1}}{2}\right)|Y|^{2}+i C_{3}
$$

where $C_{3}$ is a real constant. This equation is equivalent to

$$
(\arg Y)^{\prime}=\frac{1}{8}|Y|^{2}+\frac{1}{4}+\frac{1}{8\left(k_{0} \varepsilon\right)^{2}}-\frac{3 C_{1}}{4}+\frac{C_{3}}{2|Y|^{2}} .
$$

Next, the derivative $\mid Y^{2^{\prime}}=Y^{\prime} Y^{*}+Y^{*} Y$ is formed from equation $(5.1 b)$, it is differentiated and the right hand side simplified from equations $(5.1 a, c)$ and equations $(5.3 a, b)$ to yield $|y|^{2 "}$ as a cubic equation in $|x|^{2}$. This may now be integrated and written

$$
\text { where } \begin{aligned}
\left.\left.|| y\right|^{2}{ }^{0}\right|^{2} & =\alpha_{0}|y|^{8}+\alpha_{1}|y|^{6}+\alpha_{2}|y|^{4}+\alpha_{3}|y|^{2}+\alpha_{4}, \\
\alpha_{0} & =\frac{7}{16}, \\
\alpha_{1} & =\frac{3}{8\left(k_{0} \varepsilon\right)^{2}}-\frac{5 C_{1}}{4}, \\
\alpha_{2} & =-\left(\frac{1}{4\left(k_{0} \varepsilon\right)^{2}}+\frac{C_{1}}{2}\right)^{2}+C_{1}^{2}-C_{2}^{2}+\frac{3}{2} C_{3} \ldots \\
\alpha_{3} & =-2 C_{3}\left(\frac{1}{4\left(k_{0} \varepsilon\right)^{2}}+\frac{c_{1}}{2}\right),
\end{aligned}
$$

and $\alpha_{4}$ is arbitrary.

Equation (5.5) defines $\tau$ as an elliptic integral of the first kind in $|Y|^{2}[13]$, which means in particular that $|Y|^{2}$ is periodic in $\tau\left(=\varepsilon^{2} t\right)$. The equation may be rearranged in particular examples to: calculate the elliptic integral solution, but instead the periodicity is demonstrated more directly by simplifying the quartic in $|y|^{2}$. If the initial values of $x, y, z$ are denoted by $x_{0}, y_{0}, z_{0}$ respectively, equation (5.5) may be rewritten

$$
\begin{aligned}
{\left[|Y|^{2}\right]^{2}=} & -\left[\left(|Y|^{2}-\left|Y_{0}\right|^{2}\right)\left(\frac{3}{4}|Y|^{2}+\frac{3}{4}\left|Y_{0}\right|^{2}-\frac{1}{4\left(k_{0} \varepsilon\right)^{2}}-\frac{C_{1}}{2}\right)\right. \\
& \left.+X_{0} Y_{0}^{* 2} Z_{0}+Y_{0}^{*} Y_{0}^{2} Z_{0}^{*}\right]^{2}+|Y|^{4}\left\{\left(|Y|^{2}-C_{1}\right)^{2}-C_{2}^{2}\right\}
\end{aligned}
$$

For the particular case when $\left|\mathrm{X}_{0}\right|=\left|\mathrm{z}_{0}\right|$ and $\arg \mathrm{X}_{0}=\arg \mathrm{X}_{0}=\arg \mathrm{z}_{0}$, 
$\mathrm{C}_{2}=0$ and equation $(5.6)$ reduces to

$$
\begin{aligned}
\left.\left.|| Y\right|^{2}\right|^{2}= & \left(|Y|^{2}-\left|Y_{0}\right|^{2}\right)\left(\frac{7}{4}|Y|^{2}+\frac{7}{4}\left|Y_{0}\right|^{2}-\frac{1}{4\left(k_{0} \varepsilon\right)^{2}}-\frac{3 C_{1}}{2}\right) x \\
& {\left[\frac{1}{4}|Y|^{4}+\left(\frac{1}{4\left(k_{0} \varepsilon\right)^{2}}-\frac{C_{1}}{2}\right)|Y|^{2}+\frac{7}{4}\left|Y_{0}\right|^{4}-\left(\frac{1}{4\left(k_{0} \varepsilon\right)^{2}}+\frac{3 C_{1}}{2}\right)\left|Y_{0}\right|^{2}\right] . }
\end{aligned}
$$

When $Y$ satisfies the steady solution (equations. 5.2 ), equation (5.7) becomes

$$
\left[|y|^{21}\right]^{2}=\frac{7}{16}\left(|y|^{2}-a_{0}^{2}\right)^{2}\left[|y|^{4}+\frac{2}{3}\left(13 a_{0}^{2}-2\right)|y|^{2}-7 a_{0}^{4} \mid\right.
$$

This equation, with $k_{0} \varepsilon=1$, is sketched in Eigure $4(a)$ as a plot of $\left[|Y|^{2}{ }^{1}\right]^{2}$ against $|Y|^{2}$. Necessary conditions for a physically relevant solution are $\left[|y|^{2}\right]^{2} \geqslant 0$ and $|Y|^{2} \geqslant 0$, both of which are satisfied at the double root $|\mathrm{Y}|^{2}=\mathrm{a}_{0}^{2}=0.2$, corresponding to the steady solution $\mathbf{Y}=\mathbf{a}_{0}$ 。

When the steady solution with $k_{0} E=1$ is disturbed by an initial perturbation of the central mode, namely $x_{0}=a_{1}, Y_{0}=a_{0}+0.1$, $z_{0}=a_{1}$, the resulting evolution is the same in essence as in the first example of the previous section (where the initial perturbation $\hat{b}$ was 0.01 instead of 0.1 here). Equation (5.7) is applicable to these initial conditions, and reduces to

$$
\left[|Y|^{2}{ }^{1}\right]^{2}=\frac{7}{16}\left(|Y|^{2}-0.3\right)\left(|Y|^{2}-\frac{13}{70}\right)\left(|Y|^{4}+0.2|Y|^{2}-0.39\right) \text {, }
$$

since $Y_{0}^{2}=0.3$. This equation is sketched in figure $4(b)$ as a plot of $\left[\left.|y|\right|^{\prime}\right]^{2}$ against $|y|^{2}$. The initial point on the graph is at $|Y|^{2}=0.3,|Y|^{2}=0,|Y|^{2} "<0$, from which $|Y|^{2}$ decreases until it reaches the point $|Y|^{2}=\frac{13}{70},|Y|^{2}=0,|Y|^{2}=0$, where $|Y|$ increases until it returns to the initial point, and so on. Hence $|Y|$ is a periodic function of $\tau\left(=\varepsilon^{2} t\right)$, with a minimum value 0.43 and a maximum value 0.55 , while from equations $(5.3 a, b)|x|$ and $|z|$ have the same period as $|y|$ with equal maxima 0.33 and equal minima 0.22 . The 
rate of change of arg $Y$ is given by equation $(5.4 \mathrm{~b})$, and in this example has a mean of about -0.1 .

The effect of a perturbation applied to the nonlincar wave group is to redistribute the quartic in figure $4(a)$ so that it has a finite intersection with the $|\mathrm{y}|^{2}$-axis in the neighbourhood of the initial point. For a small initial perturbation, the change in height of the quartic near the initial point is sma11, but because of the flat slope of the quartic near this point, the range of $|Y|$ in the resulting periodic motion may be much larger. However, the important property is that for any small perturbation of the three wave mode nonlinear wave group, the resulting evolution of the wave group is strictly periodic, even though a linear stability analysis shows that the wave group is unstable to small perturbations.

\section{Numerical solution of the evolution equations}

The evolution equation for $B_{k}$ (equation 4.2 ) may be rewritten as the difference equation

$$
\begin{aligned}
\frac{B_{k}(\tau+\Delta \tau)-B_{k}(\tau-\Delta \tau)}{2 \Delta \tau} & -i P_{k} \frac{B_{k}(\tau+\Delta \tau)+B_{k}(\tau-\Delta \tau)}{2} \\
& =i \sum_{\ell} \sum_{m} R_{k l m} B_{\ell}^{*}(\tau) B_{m}(\tau) B_{k+l,-m}(\tau),
\end{aligned}
$$

where $\tau=\varepsilon^{2} t$, and this equation then used to generate a stepwise numerical solution of $B_{k^{\prime}} k_{0}-k_{1} \leqslant k \leqslant k_{0}+k_{1}$. This form of difference equation was applied successfully to quadratic nonlinearities in a previous investigation [14], and is such that the linear terms are numerically stable. The time step used in most calculations is $\Delta \tau=\pi / 200$. The numerical solution described in $\$ 4$ and in Figure 3 was calculated by this method. 
The princtpal example to be described bore i:s is generalisition of the 3 wave mode example solved analytically in thr provious section. The system 1 extended here to 21 wave modes, $90 \leqslant \mathrm{k} \leqslant 110$, and the calculations are started from the same initial conditions as before; namely $B_{100}=a_{100}+0.1, B_{k}=a_{k}$ otherwise: The results are presented in figure 5.

The moduli of $B_{99}, B_{100}$, and $B_{101}$ axe drawn in figure $5(a)$. The important property of this solution is that the moduli are found to be almost periodic over the 5000 time steps of the integration, with no evidence of a loss of periodicity beyond the interval of calculation. The form of the solution agrees with that found in the last section for a 3 wave mode system, but the values for the amplitude and the period of the moduli differ fxom those calculated because of the influence of the additional 18 wave modes.

The arguments of $\mathrm{B}_{99}, \mathrm{~B}_{100}$, and $\mathrm{B}_{101}$ are drawn in figure $5(\mathrm{~b})$. The interesting feature here is that the negative mean cate of change of argument is nearly the same for all three wave modes, indicating that the phase velocity of the wave inside the wave group is increased as a result of the initial positive perturbation of the central wave mode. Also, since the phase difference between the wave modes remains almost zero, the change in envelope of the wave group is detexmined almost solely by the change in moduli of the wave modes.

The upper side of the wave group envelope is drawn in figure 5 (c) as a function of $x$ and $\varepsilon^{2} t$, relative to an observer moving with the propagation velocity $\frac{1}{2}$ of the unperturbed wave group. The intervil if slow time that is illustrated is $0 \leqslant \varepsilon^{2} t \leqslant 15 \pi$, only part of that in figures $(5 a, b)$. It can be seen that the small oscillation in shape of the envelope is related directly to the periodic oscillation of the moduli. This figure illustrates one of the main points made by this 
investigation, that the calculated lincax instability of the nonlinear wave group gives a false impression of the evolution of the wave group. The behaviour of the wave group shown in this figure is the behavioux of a stable system, and the definition of stability that is applied to this systern should be consistent with this figure.

A comparison of figures $3(a)$ and $5(a)$ shows that the period of oscillation of the moduli, like their amplitudes, is dependent on the magnitude of the initial perturbation. This is to be expected for a nonlinear system such as this, and is demonstrated by the analytical solution in $\$ 5$. 


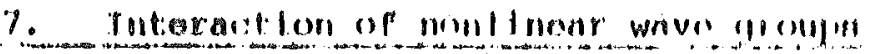

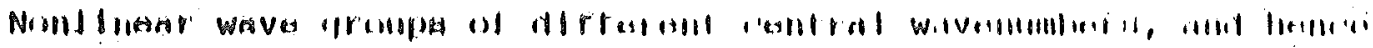
of different propagation velocities, have beon shown theoretically and experimentally $|8|$ to have the soliton property that when they interact, they emerge from the interaction with at most a change of phase. The numerical method described in the previous section is applied here to the calculation of the interaction between nonlinear wave groups of the same and of almost the same central wavenumbers propagating in the same direction.

The three wave mode analytical solution (\$5) may be applied to finding the values that should be given to the amplitudes and phases of two nonlinear wave groups of the same central wavenumber so that the interaction between them is as large as possible. Following these approximate calculations, the initial conditions were chosen to describe a nonlinear wave group for which $\varepsilon=0.01, k_{0}=100$, superposed on a second nonlinear wave group for which $\varepsilon=0.0075, k_{0}=100$, with their envelope crests at half the fundamental wavelength apart, and their central wave modes in phase. The evolution of this system, calculated for the waveband $90 \leqslant k \leqslant 110$ over the interval $0 \leqslant \varepsilon^{2} t \leqslant 15 \pi$, is sketched in figure 6 .

The moduli of $B_{99}, B_{100}$, and $B_{101}$ in figure $6(a)$ display excellent periodicity over the interval calculated, even through the amplitudes of oscillation are much larger than in previous examples. The complex amplitude $B_{100}$ passes through zero where there is a discontinuity of $\pi$ in its argument. The evolution of the two envelopes is illustrated in figure $6(\mathrm{c})$, where it can be seen that the interaction between the two nonlinear wave groups has the form of an oscillation of energy between them, without any significant change in their relative positions. The final example calculated has the same initial conditions as in the previous example except that the central wavenumber of the second 
nonlinear wave gxoup is $k_{0}=98$. Ihe paths of the envelopes ot the two wavetraing may br: discrened in tho upper envelope surface in firfure.7(c). The fanter nonlinear wave groups with central wavenumber 100 have moan paths parallel to the $\mathrm{C}^{2} \mathrm{t}$-axis, indicating that they are propagating with the velocity $\frac{1}{2}$ of undisturbed nonlineax wave groups at this central wavenumber. The slower nonlinear wave groups with central wavenumber 98 have mean paths across the figure, with an exchange of roles occurring between envelope crests as the two sets of crests intersect. This Figure bears a striking resemblance to that describing the interaction of two wavetrains in shallow watex ([14], figure 4), even though the present figure describes envelopes on deep water and the previous figure described waves on shallow water. The immediate reason for the resemblance is the similarity between the soliton properties of the cubic Schrödinger equation and the soliton properties of the Rorteweg and de Vries equation. A unified approach to the complete spectrum of nonlinear water waves may find deeper reasons for this resemblance. The moduli and arguments of $\mathrm{B}_{98}, \mathrm{Bg}_{9}$, and $\mathrm{B}_{100}$ are sketched in figures $7(a), 7(b)$ respectively, where it can be seen that the apparent periodicity of figure $7(c)$ is not consistent with a closer scrutiny of the wave modes at the centre of the interaction. The reasons for the loss of periodicity in this example, compared with the good periodicity in previous examples, are not known. 


\section{Discussion}

The andysde here tho been restricted to wave structures composed of wave modes in a narrow waveband, for which tertiary interactions between the wave modes are significant. Permanent wave structures of this type were found to be linearly unstable, but to be stable in a wider sense in that they exhibited a periodicity or cyclic recurrence over longer times. These properties have been demonstrated also for a modulated stokes wavetrain [9], because the modulated wavetrain was found experimentally to evolve into a 'pulse train' of the type investigated here. An attempt will be made now to apply the methods of the present investigation to modulated stokes wavetrains, for which the narrow waveband assurption does not apply initially, although the cited experiments suggest that it may apply at later times. Stokes waves are linearly unstable to sideband nodulations in the same directic as the wavetrain on deep water, but are linearly stable to the same modulations on shallow water. It is intended therefore to investigate the cyclic recurrence of nonlinear water waves for the whole range of water depths. 


\section{Leferencess}

1. D.J. Benney and A.C. Newe11, The propaqation of norlinear wave envelopes, J. Math. Phys. 46, 133-139 (1967).

2. Bruce I. Cohen, Kenneth M. Watson, and Bruce J. West, some properties of deep water solitons, Phys. Fluids. 19, 345-354 (1976).

3. V.E. Zakharov, stability of periodic waves of finite amplitude on the surface of a deep Eluid, J. Appl. Mech. and Tech. Phys. 2. $190-194$ (1968).

4. Vincent $\mathrm{H}$. Chu and Chiang C. Mei, The nonlinear evolution of Stokes waves in deep water, J. Fluid Mech. 47, 337-351 (1971).

5. Hidenori Hasimoto and Hiroaki Ono, Nonlinear modulation of gravity waves, J. Phys. Soc. Japan 33, 805-811 (1972).

6. A. Davey, The propagation of a weak nonlinear wave, J. Fluid Mech. $53,769-781(1972)$

7. G.B. Whitham, Linear and Nonlinear Waves, wiley, 1974.

8. Henry $C$. Yuen and Bruce M. Lake; Nonlinear deep water waves: Theory and experiment, Phys. Fluids. 18, 956-960 (1975).

9. Bruce M. Lake, Henry. C. Yuen, Harald Rungaldier and Warren E. Ferguson, Nonlinear deep water waves: Theory and experiment. Part 2. Evolution of a continuous wave train, J. Fluid Mech. 83, 49-74 (1977).

10. G. Roskes, Comments on "Nonlinear deep water waves: Theory and

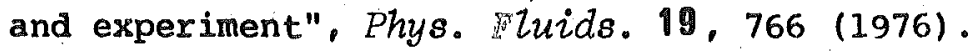


11. T. Brooke Benjamin and J.E. Feir, The disintegration of wavetrains on deep water. Part 1. Theory, ef, Fluid Mech. 27, 417-\$30 (1967).

12. P.J. Bryant, Stability of periodic waves in shallow water, J. Fluid Mech. 66, 81-96 (1974).

13. L.M. Milne-Thomson, Elliptic integrals, Handbook of Mathematical Functions, Chap 17, Ed Milton Abramowitz and Irene A. Stegun, Dover, 1965.

14. P.J. Bryant, Periodic waves in shallow water, J. Fluid Mech. 59, $625-644$ (1973). 


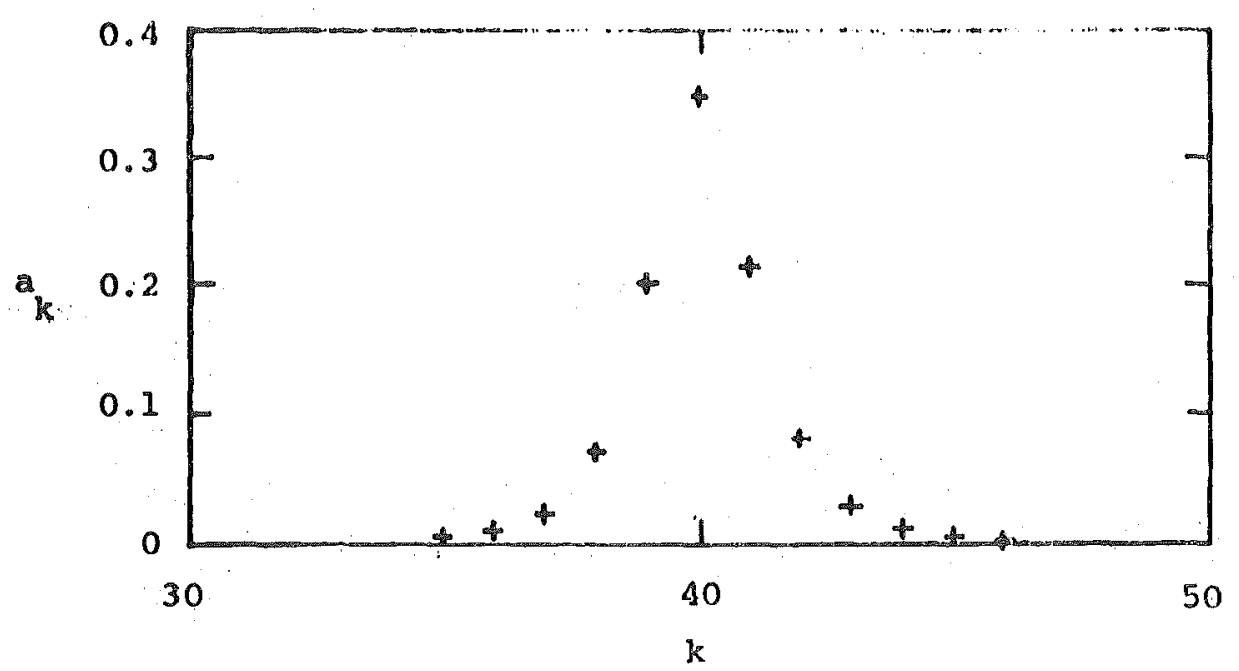

Figure $1(a)$. Wave amplitudes when $k_{0}=40, c=1 / 40$.

Figure $1(b)$. One group wavelength (horizontal contraction $64 \pi$ ) when $k_{0}=40, \varepsilon=i / 40$.

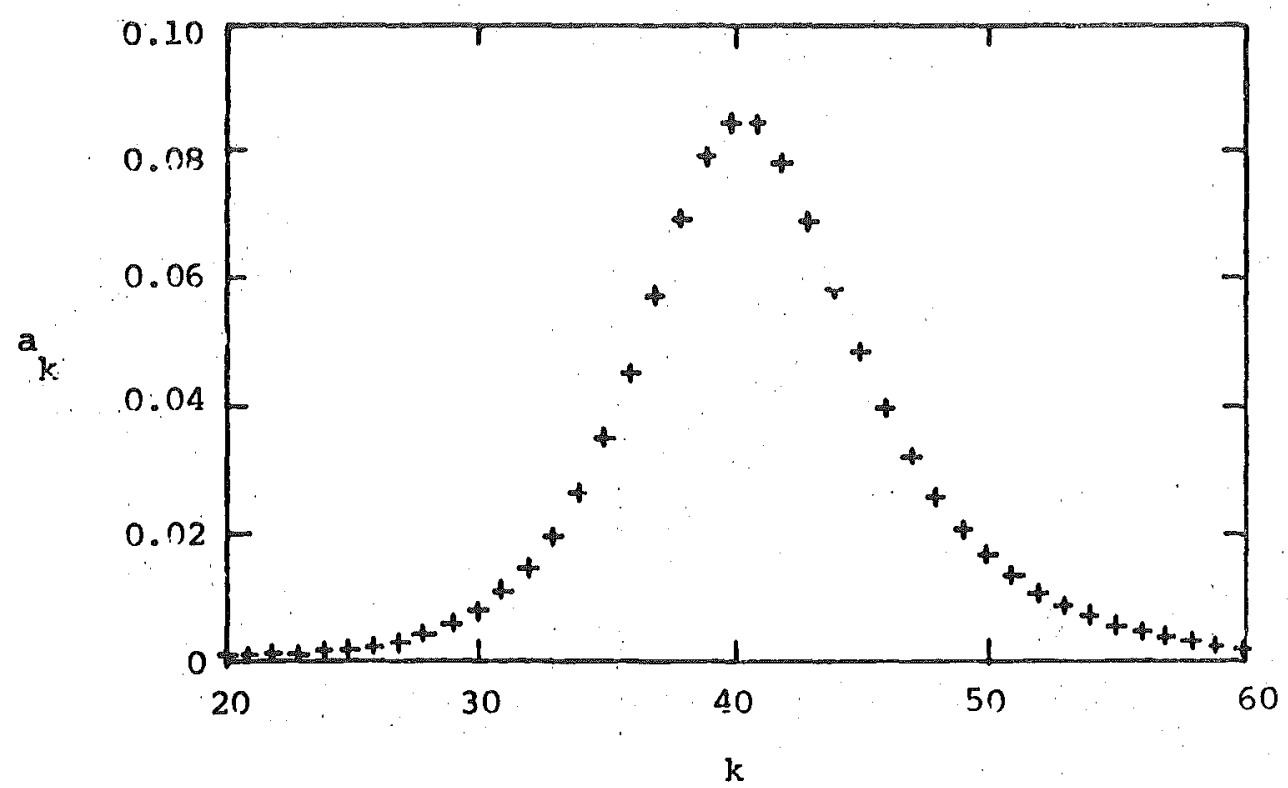

Fioure $2(a)$. Wave amplitudes when $k_{0}=40, \varepsilon=1 / 10$.

Figure $2(\mathrm{~b})$. One groun wavelength (horizontal contraction $16 \pi$ ) when $k_{0}=40, \varepsilon=1 / 10$. 

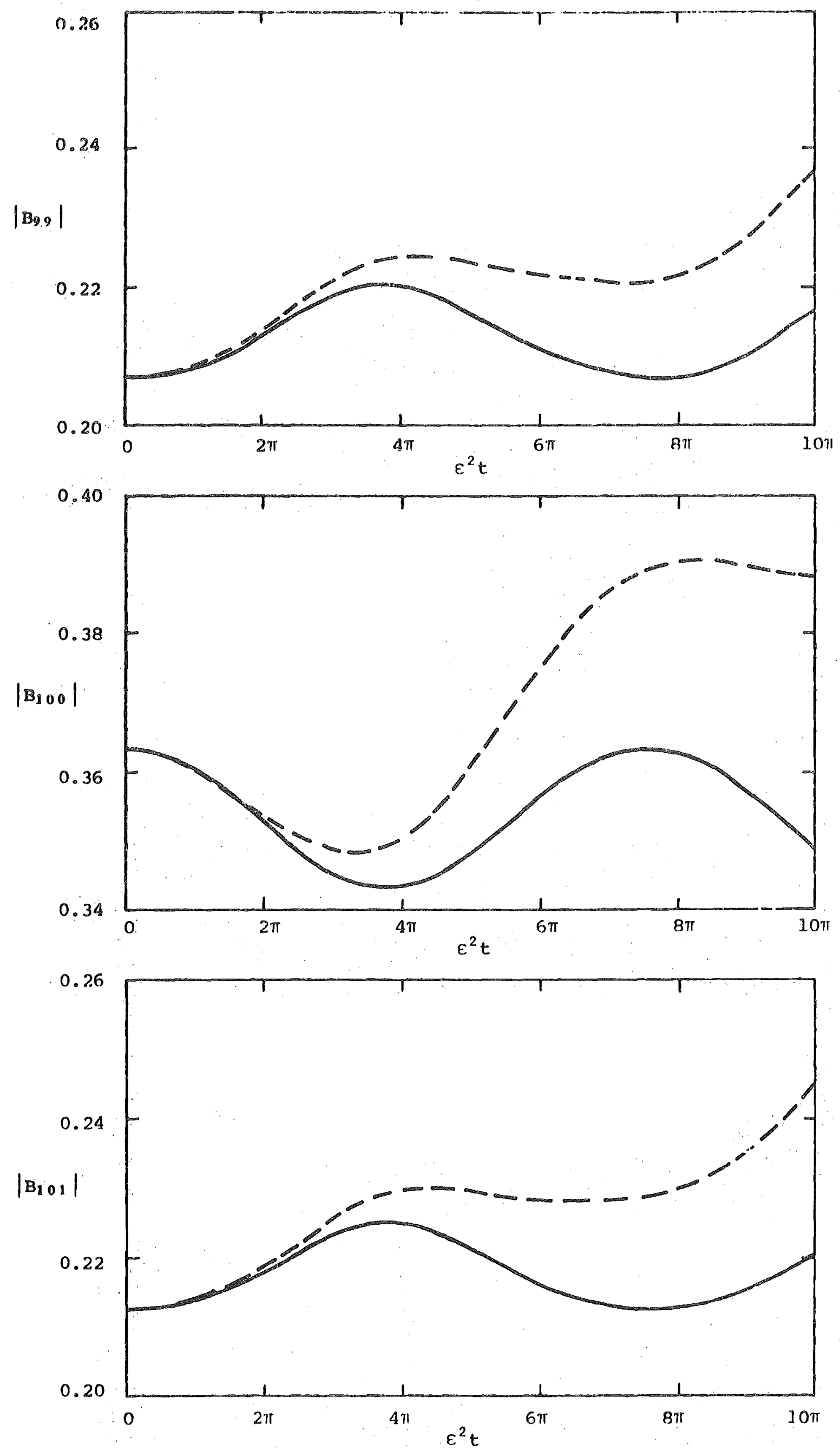

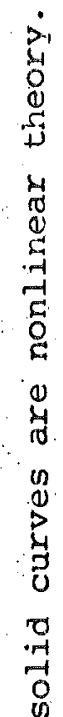



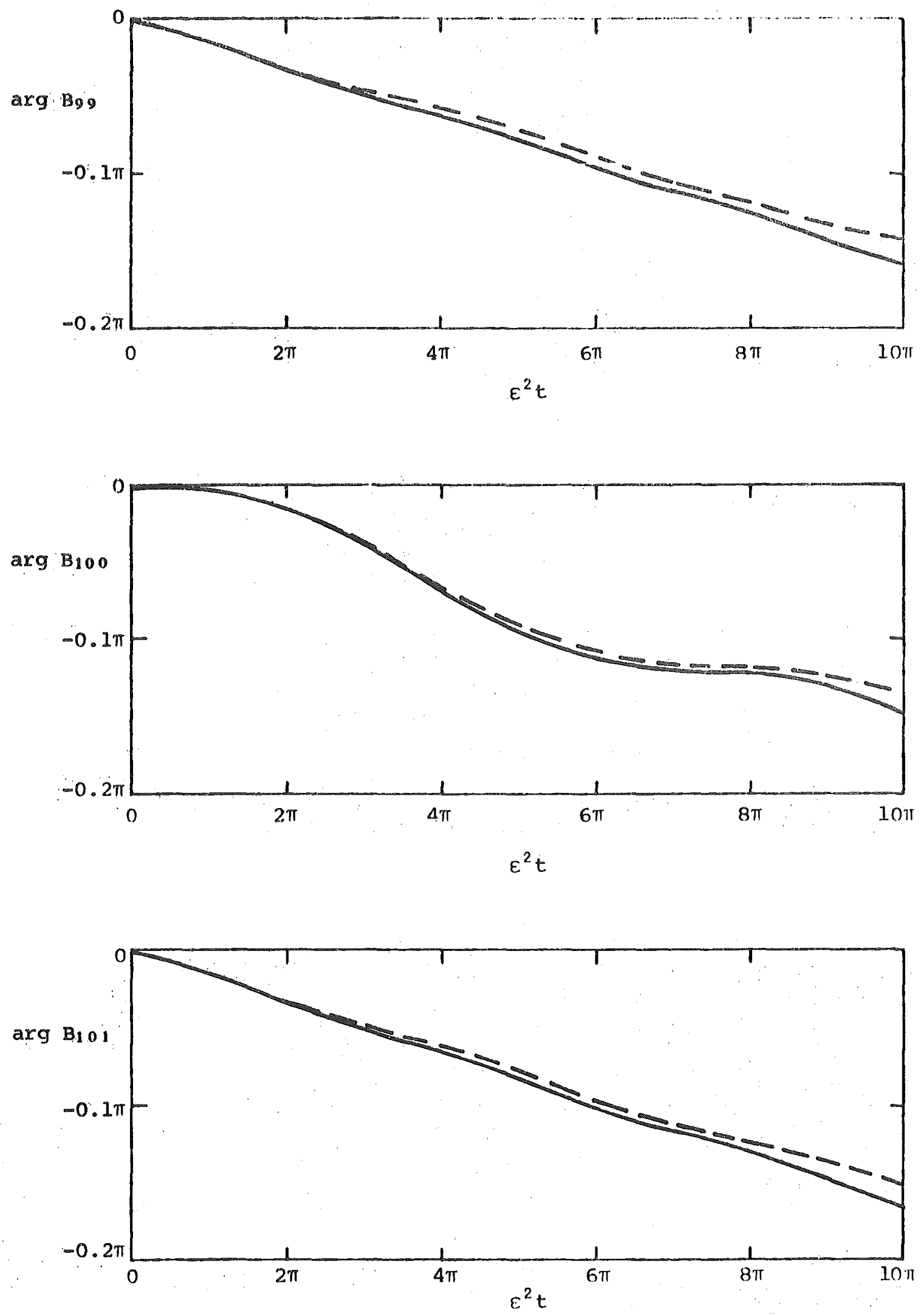

Figure $3(b)$. Arguments when $\varepsilon=0.01, k_{0}=100, k=100, \hat{b}=0.01$. 


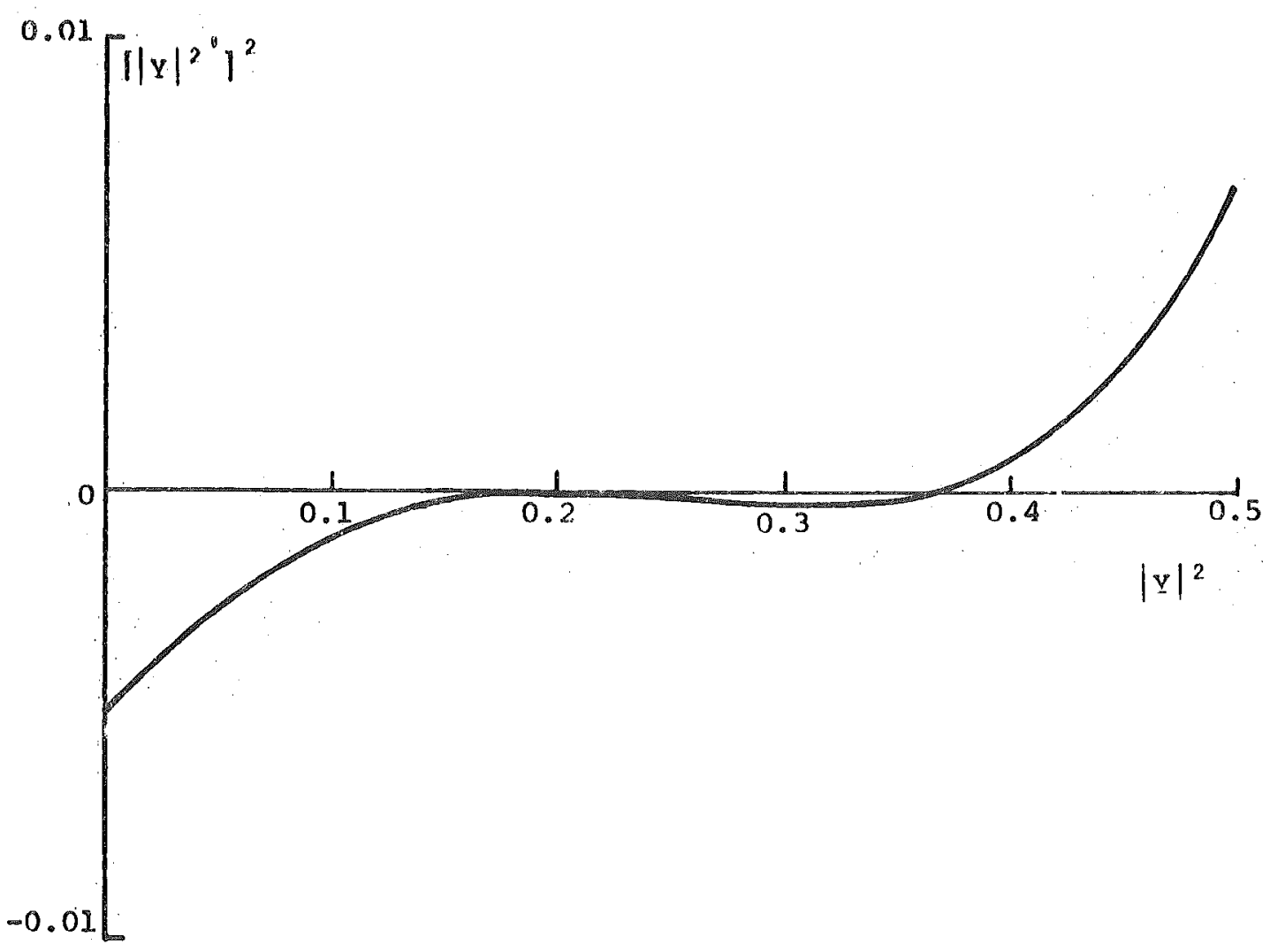

Fiquxe 4(a). Plot of equation (5.8).

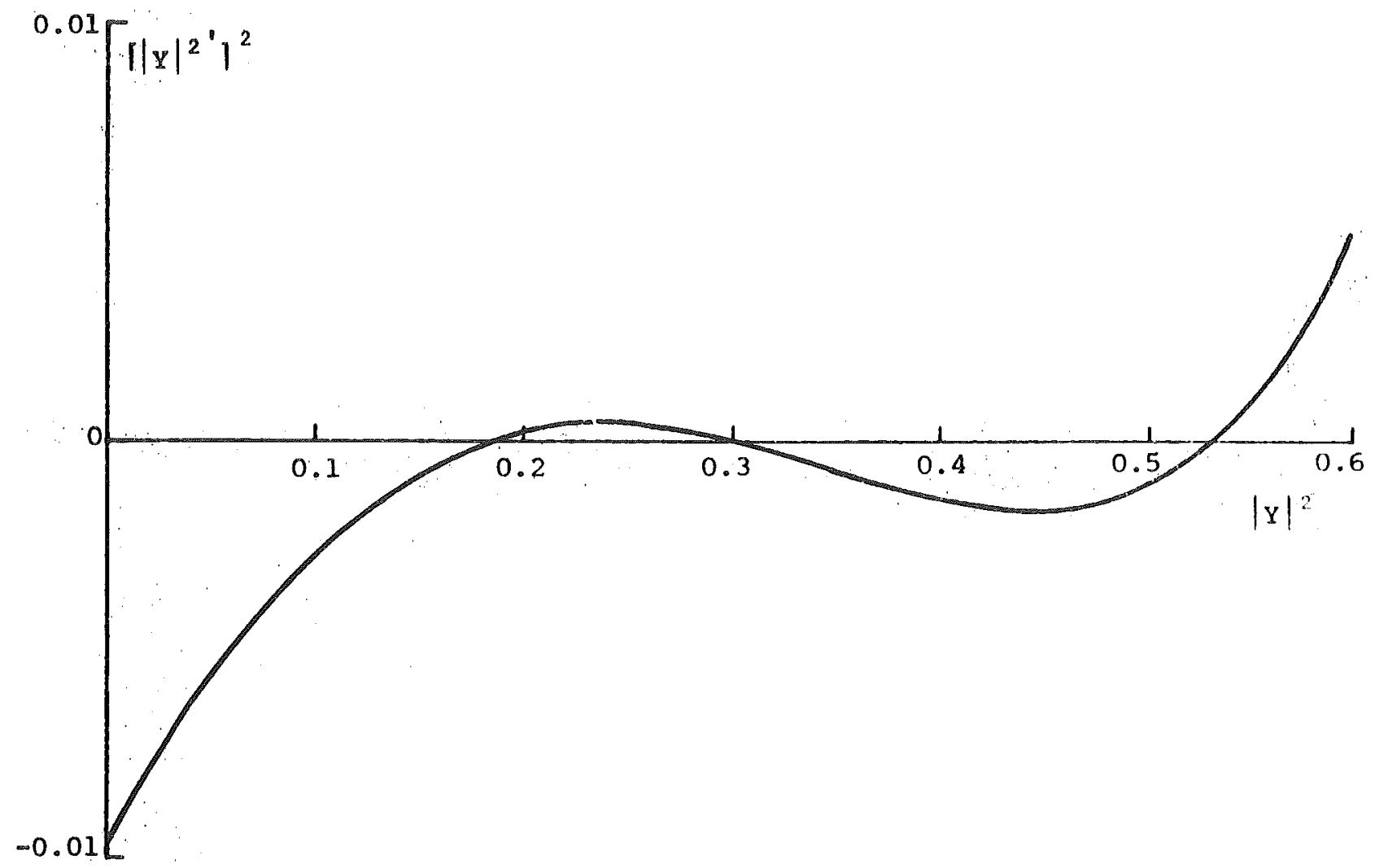

Figure $4(\mathrm{~b})$. Dlot of equation $(5.9)$ 

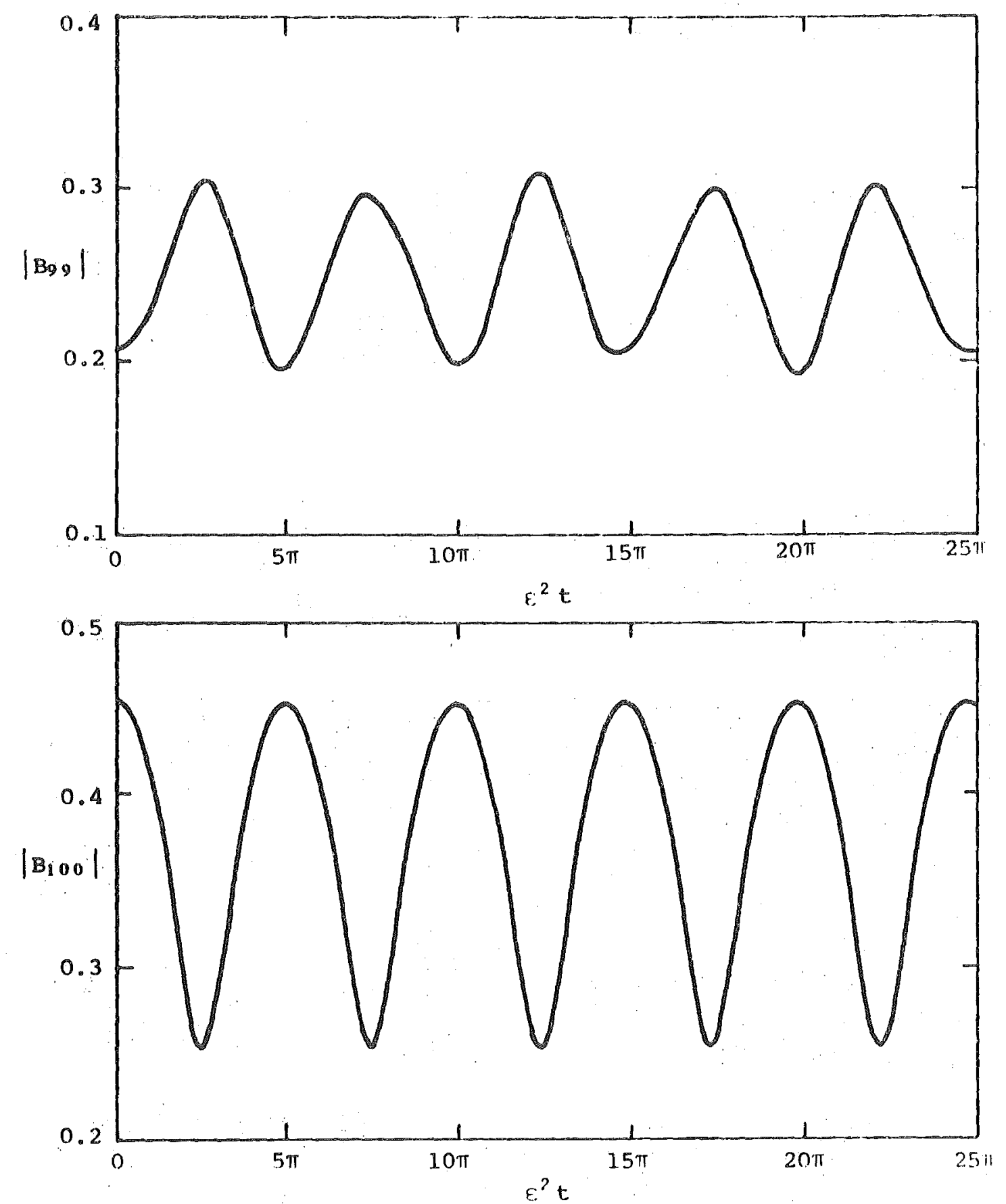

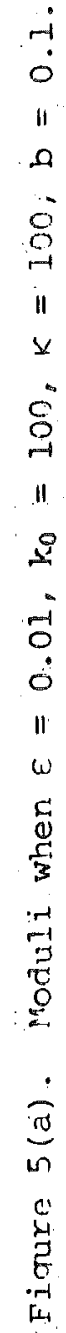

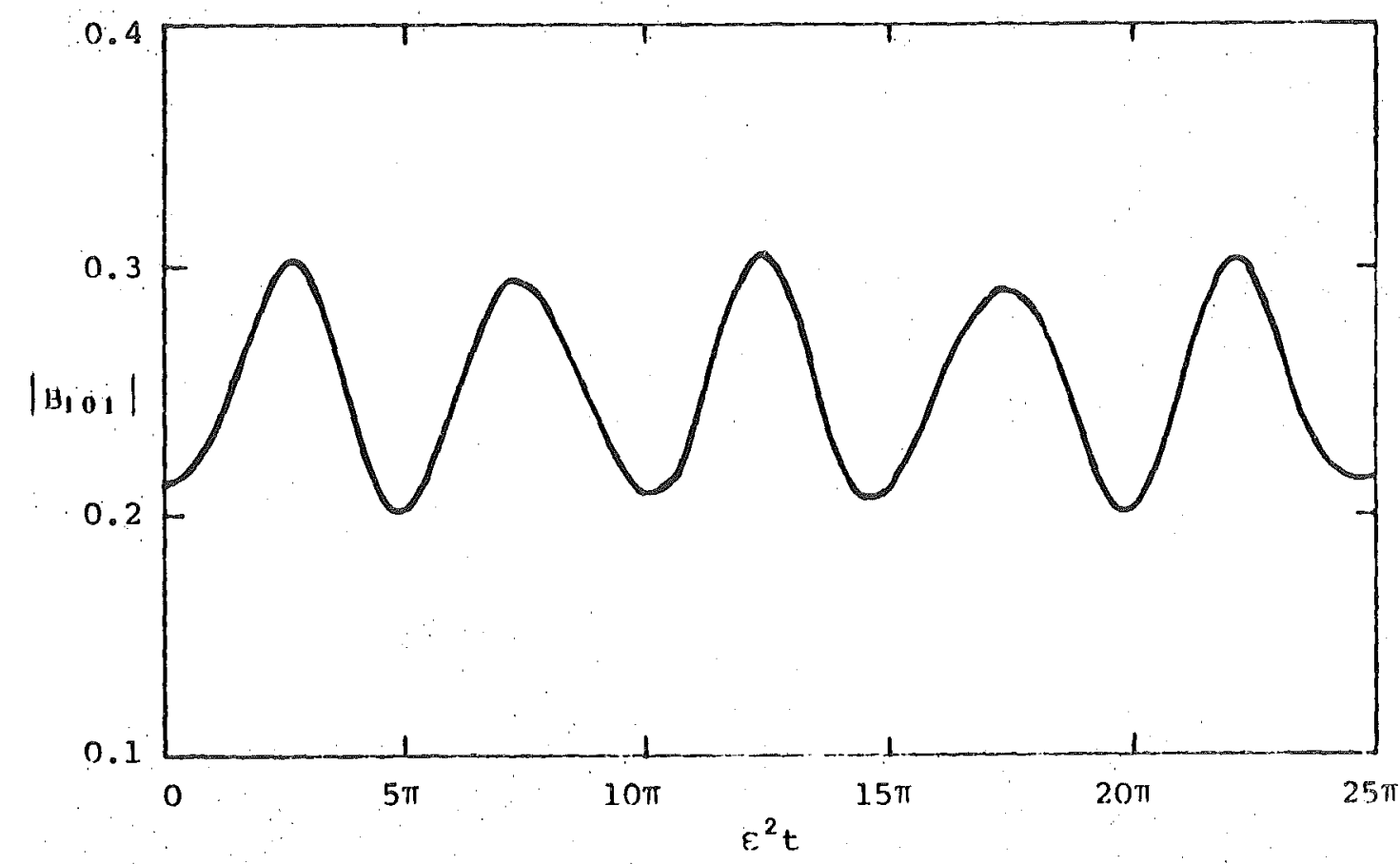



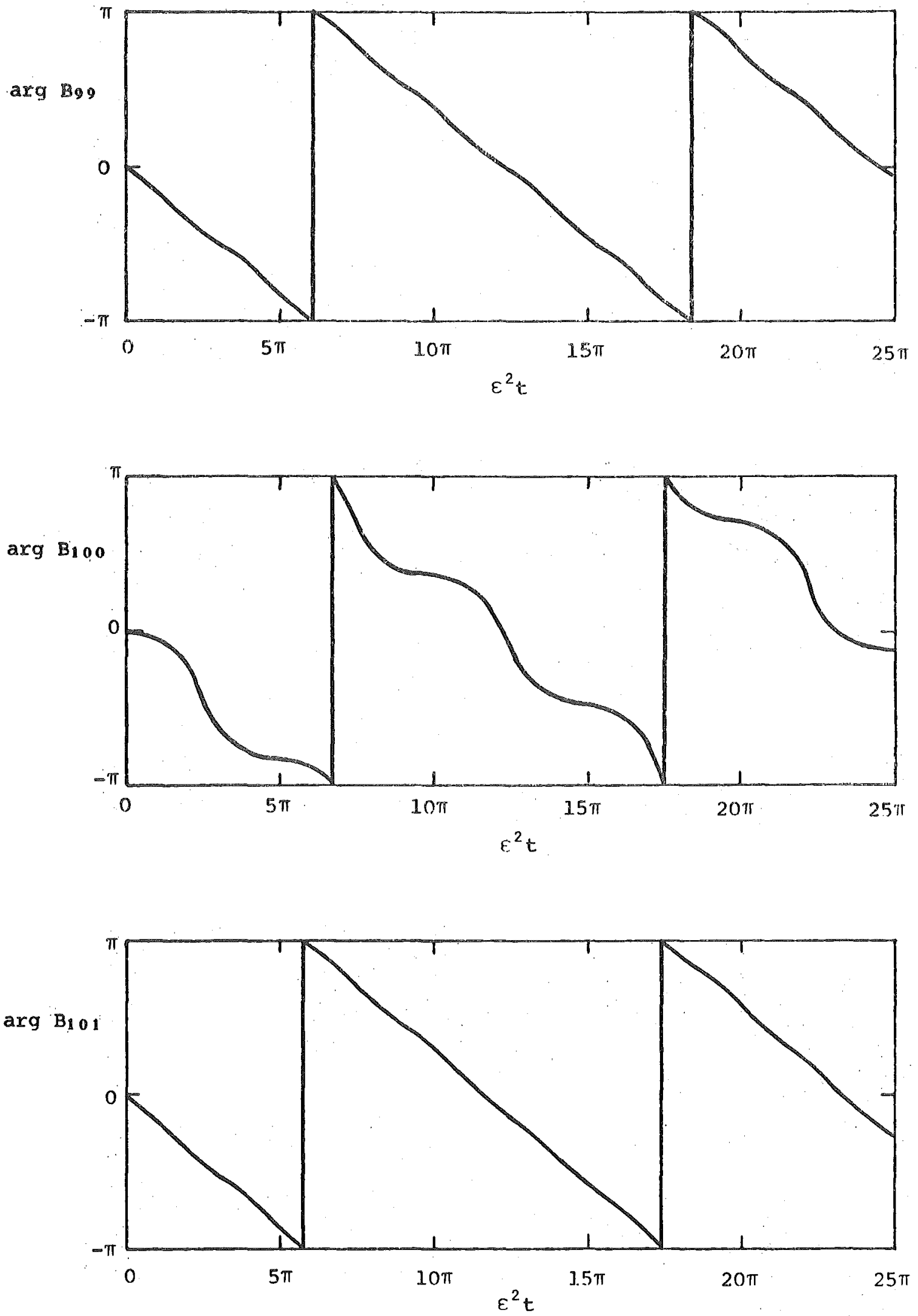

Figure 5(b). Arguments when $\varepsilon=0.01, k_{0}=100, k=100, \hat{b}=0.1$ 


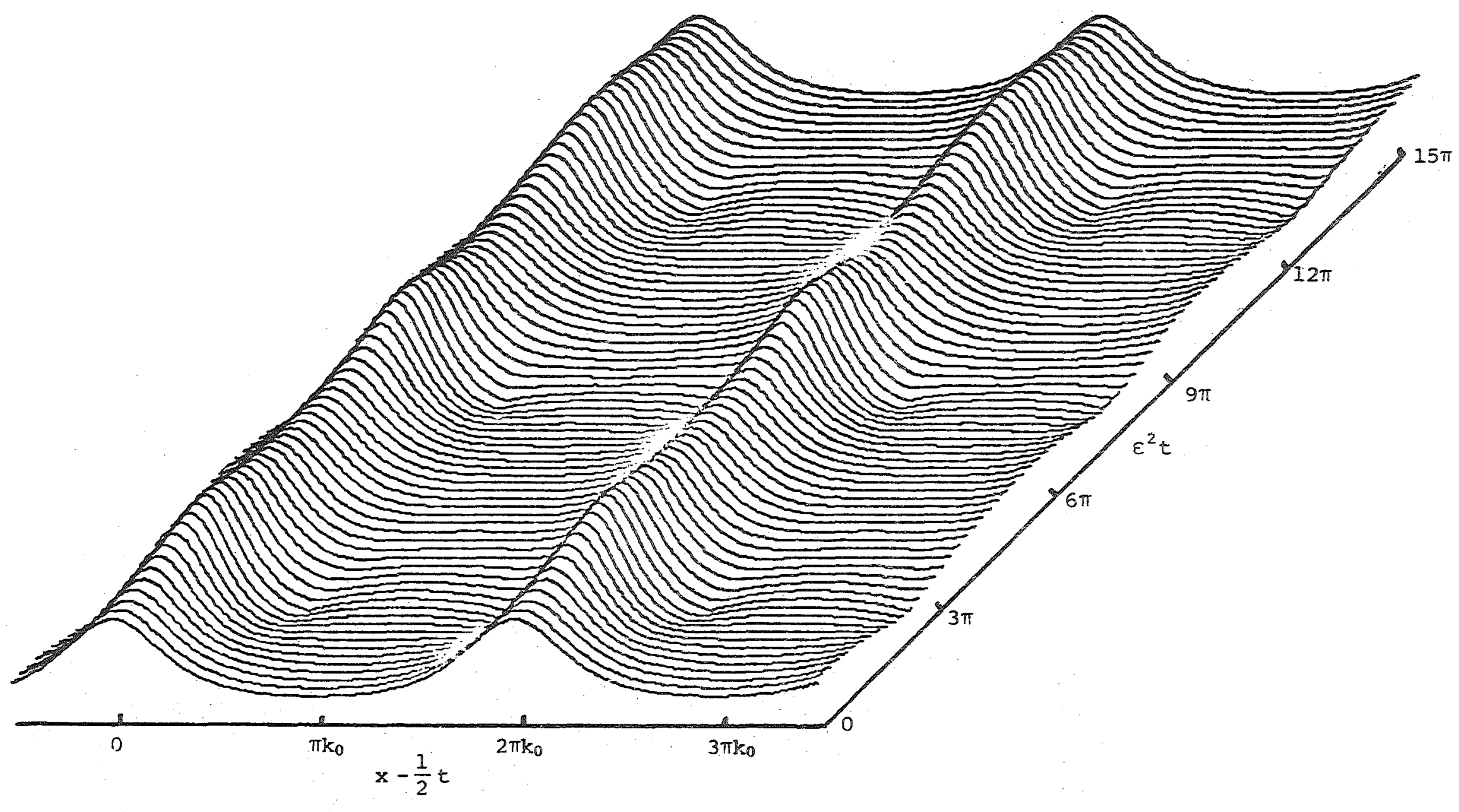

Fiqure $5(c)$. Upper envelope surface when $\varepsilon=0.01, k_{0}=100, k=100, \hat{b}=0.1$.

Horizontal contraction $4000 \pi$. 


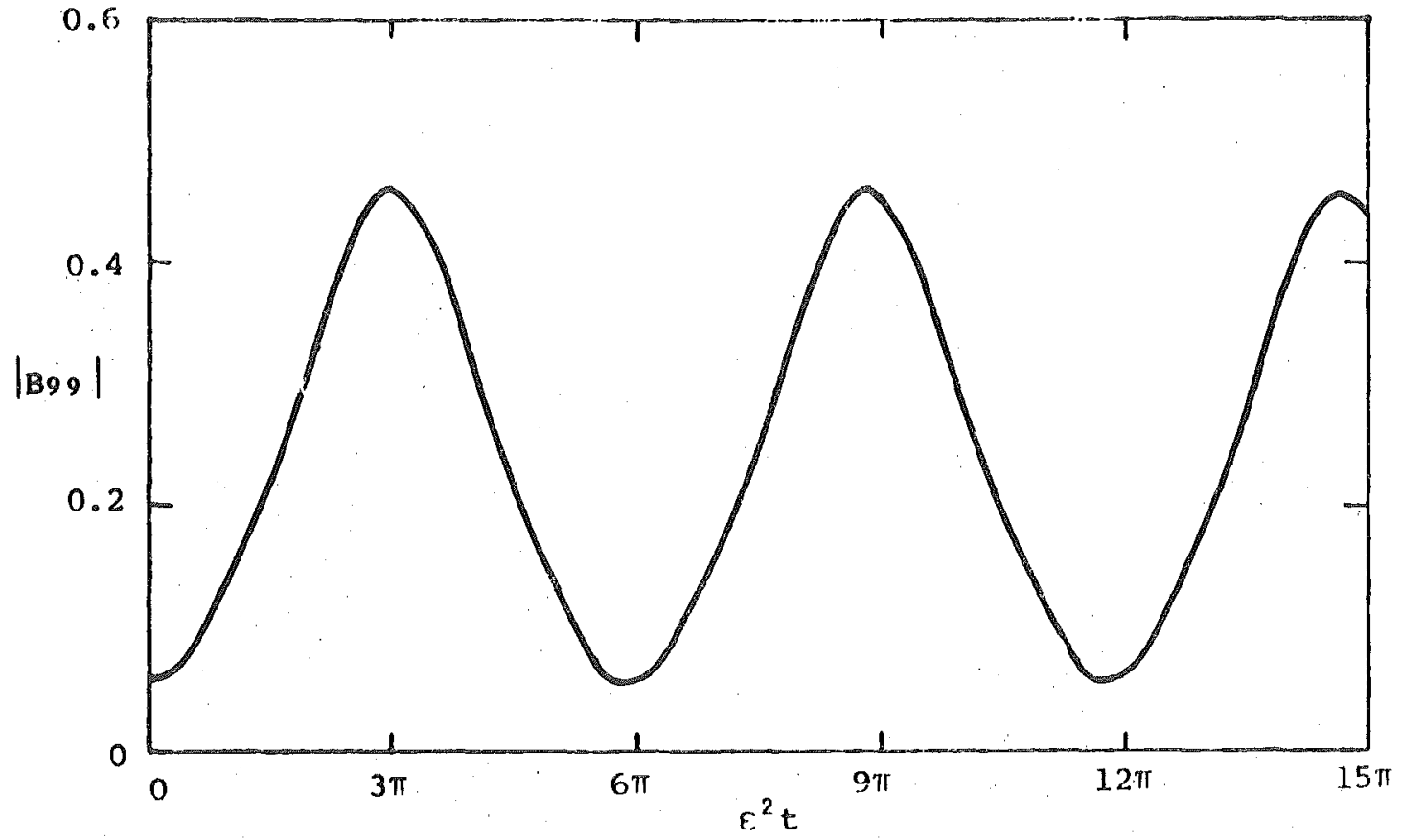

35.

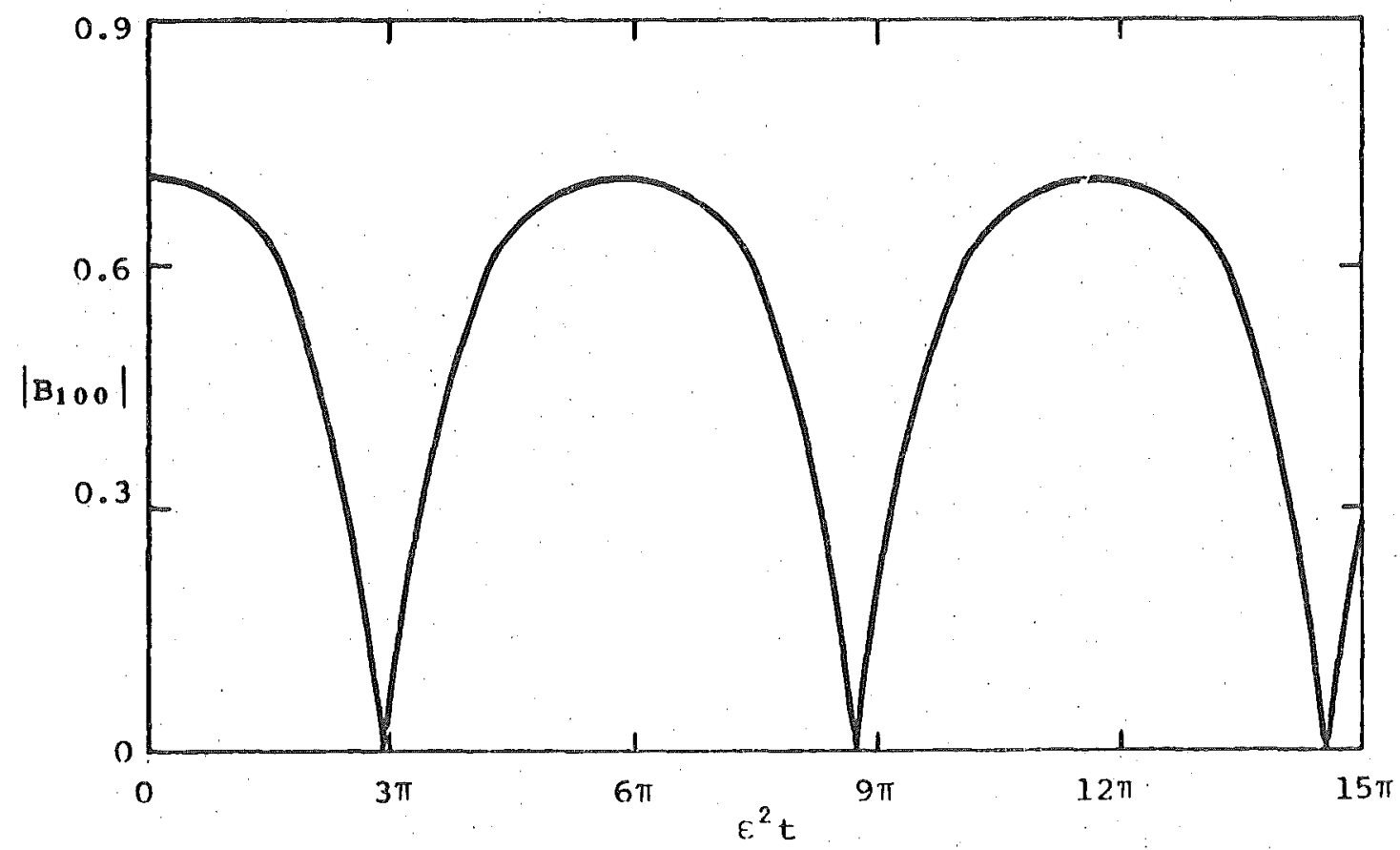

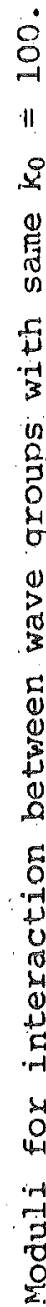

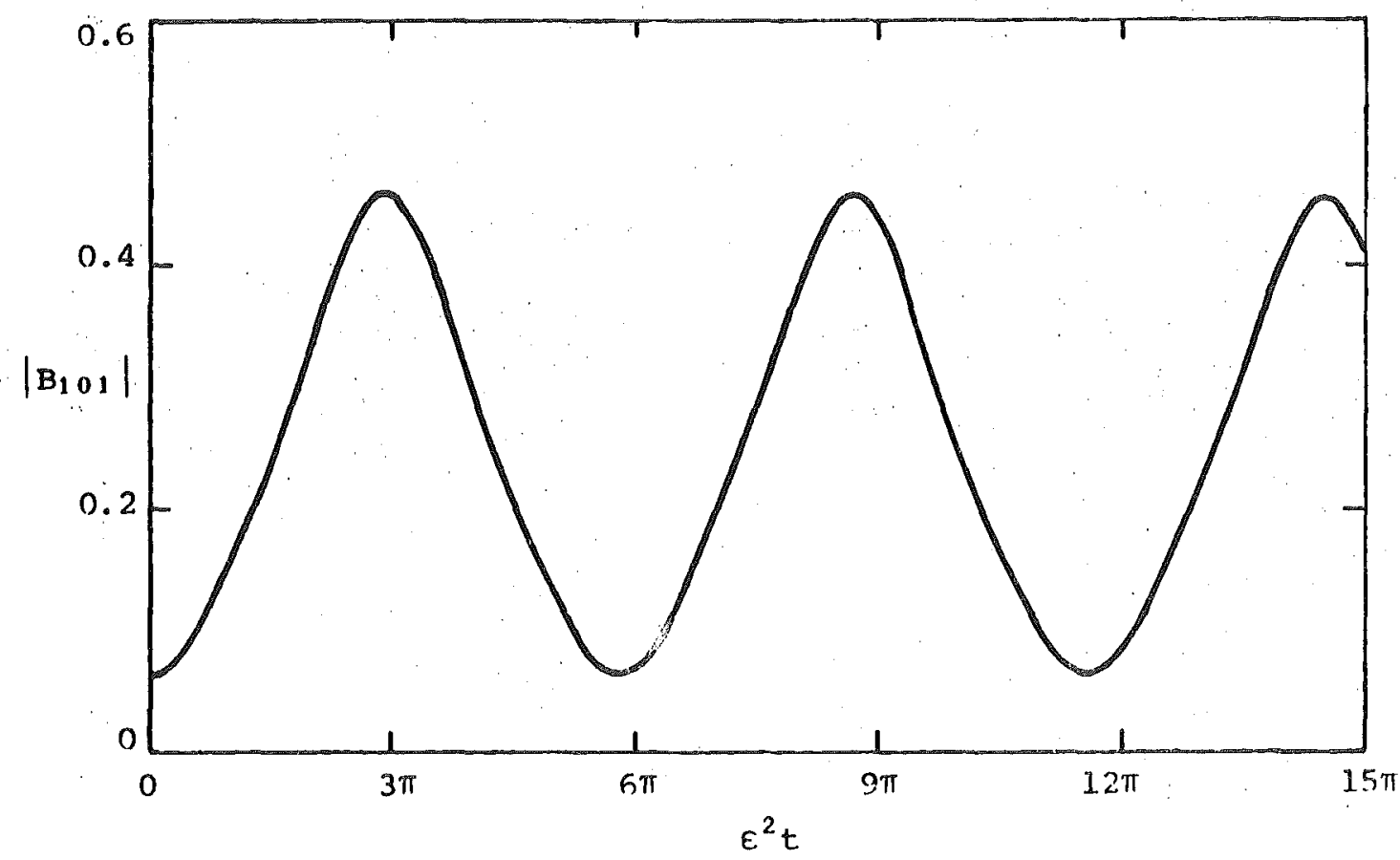


36.
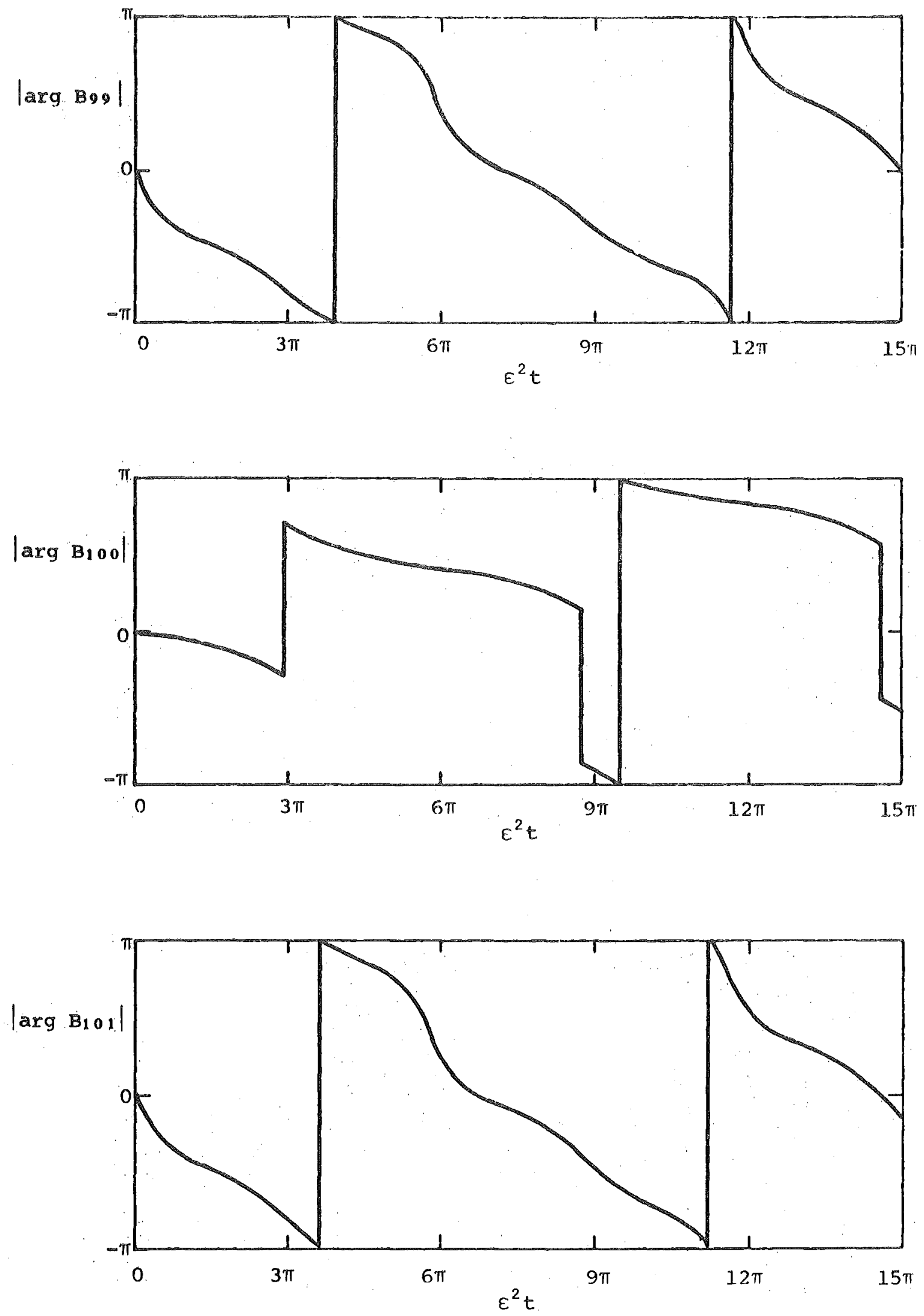

Figure $6(b)$. Arguments for interaction between wave groups with same $k_{0}=100$. 


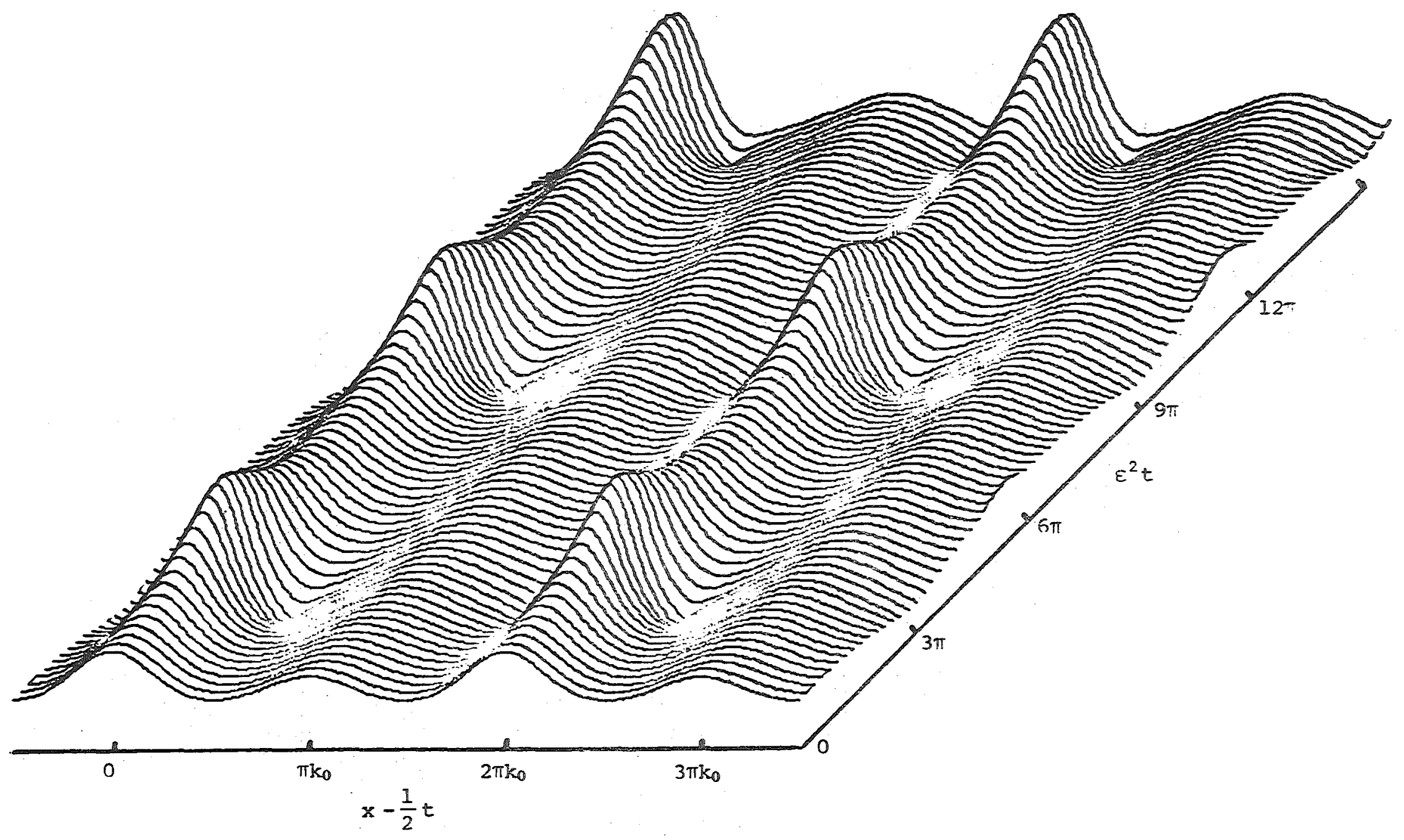

Figure $6(\mathrm{c})$. Upper envelope surface for interaction between wave grouns with same $k_{0}=100$ Horizontal contraction $4000 \pi$. 

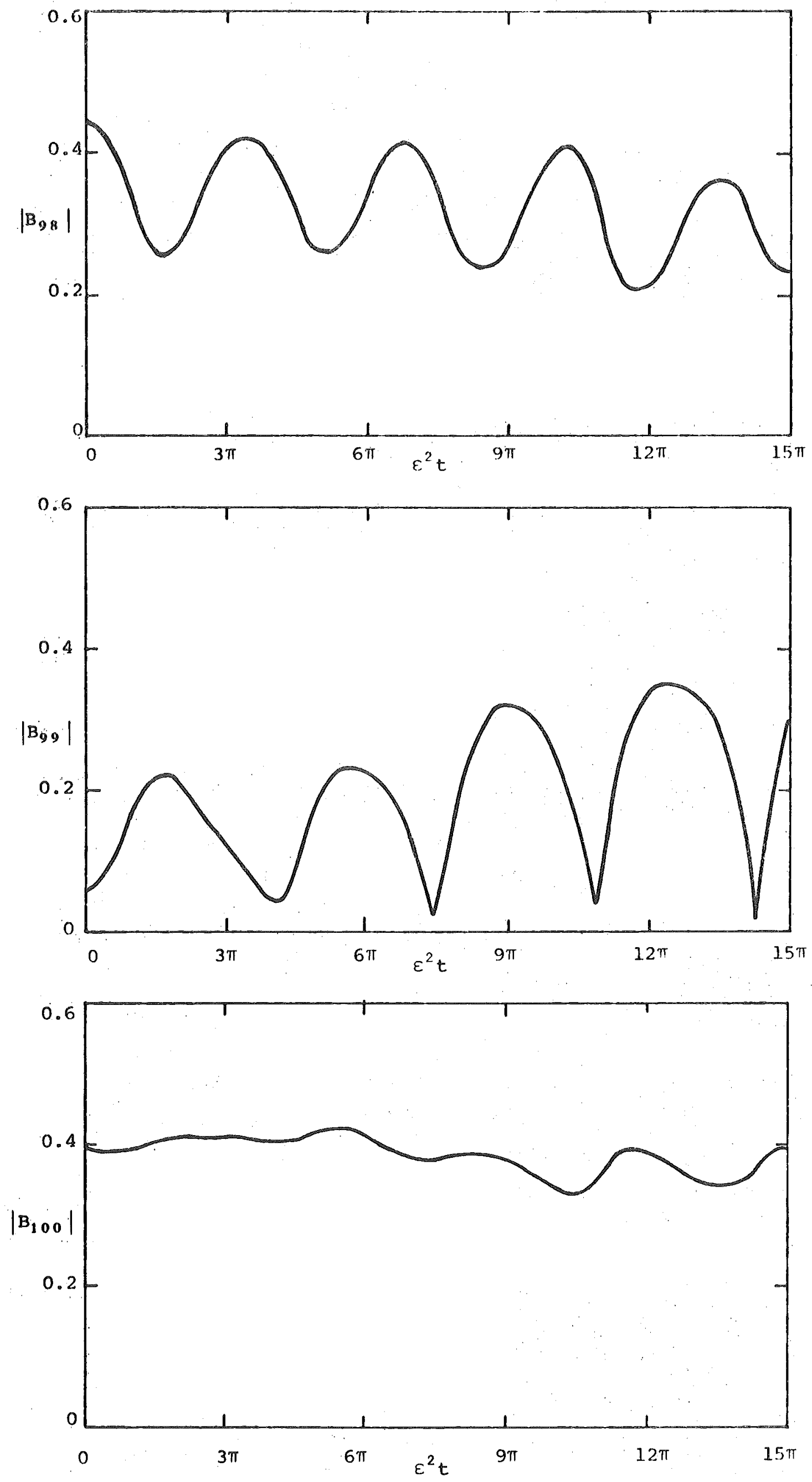

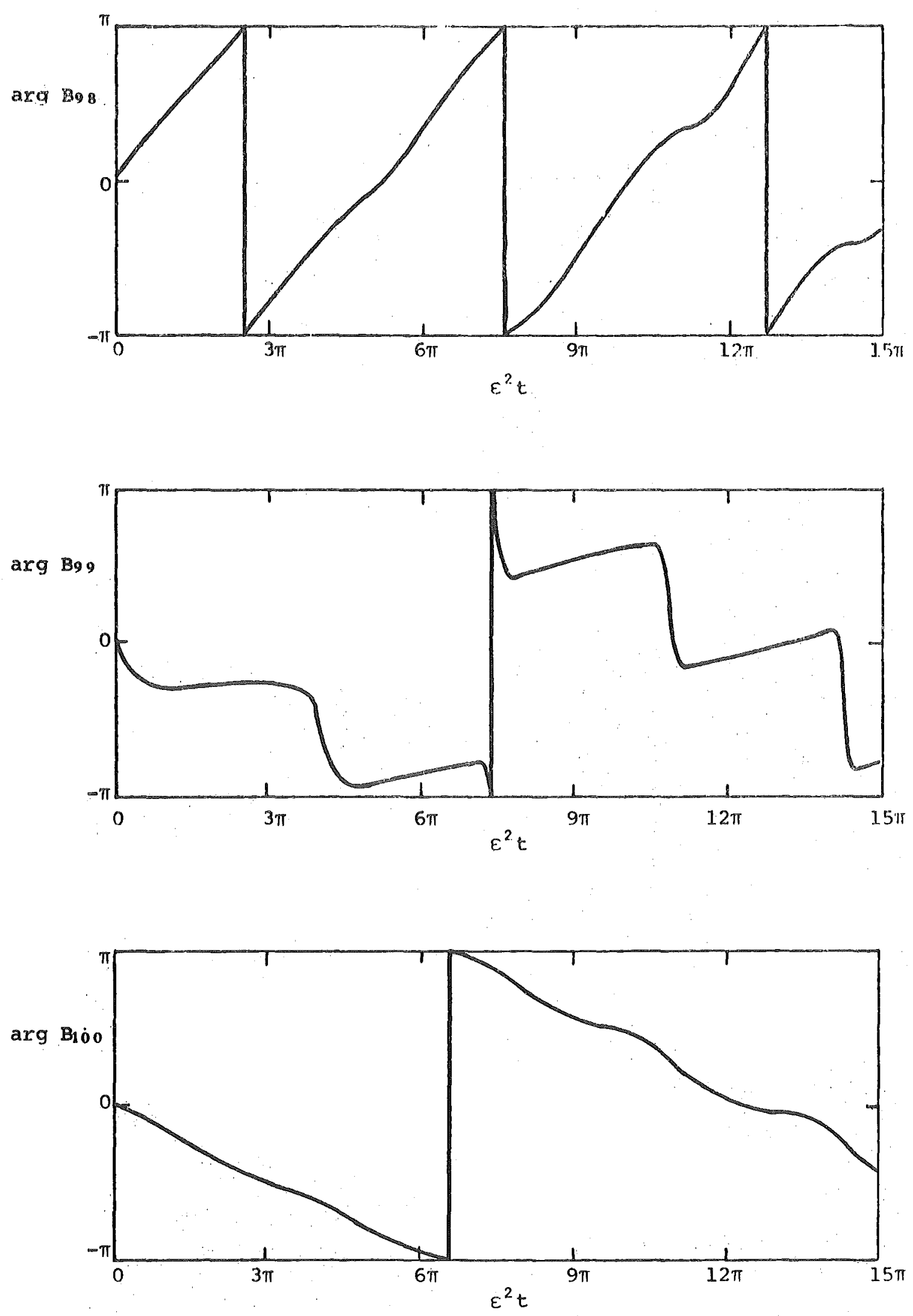

Figure $7(b) . \quad$ Arguments for interaction between wave grouns with $k_{0}=100$ and $k_{0}=98$. 


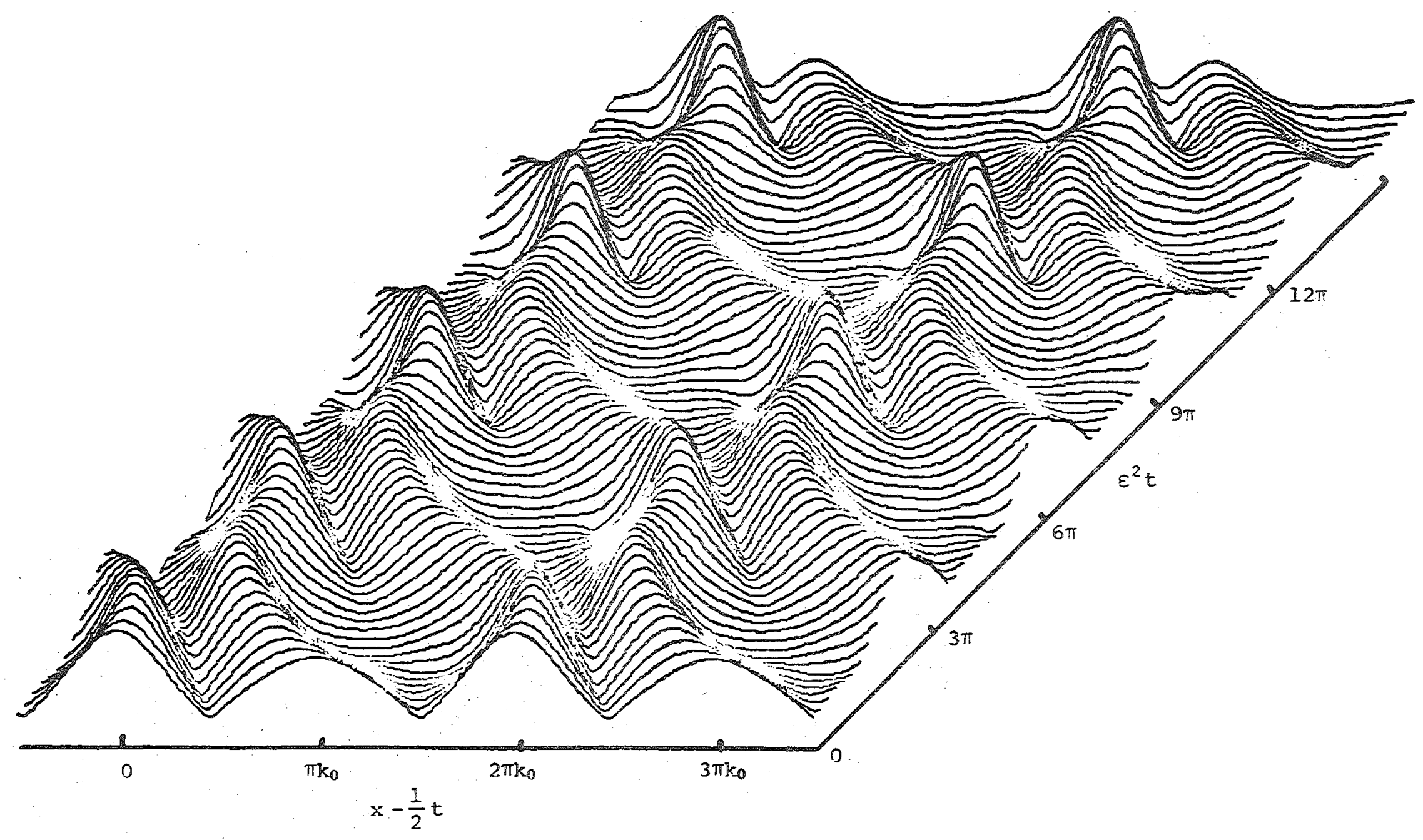

Figure $7(c)$. Upper envelope surface for interaction between wave groups with central wavenumbers 100 and 98. Horizontal contraction $4000 \pi$. 
1. (November 1976) BRYANT, P.J. (Reprinted March 1978)

Permanent wave structures on an open two layer fluid.

2. (December 1976) WEIR, G.J.

Conformal killing tensors in reducible spaces.

3. (January 1977) WEIR, G.J. \& KERR, R.P. Diverging type-D metrics.

4. (February 1977) KERR, R.P. \& WILSON, W.B.

Singularities in the Kerr-Schild metrics.

5. (MaY 1977) PETERSEN, G.M.

Algebras for matrix limitation.

6. (August 1977) GRAY, A.G.

Topological methods in second order arithmetic.

7. (September 1977) WAYLEN, P.C.

Green functions in the early universe.

8. (November 1977) BEATSON, R.K.

The asymptotic cost of Lagrange interpolatory side conditions in the space $C(T)$.

9. (November 1977) BEATSON, R.K.

The degree of monotone approximation.

10. (November 1977) BREACH, D.R.

On the non-existence of $5-(24,12,6)$ and $4-(23,11,6)$ designs.

11. (February 1978) BRYANT P.J. \& LAING A.K. Permanent wave structures and resonant triads in a layered fluid.

12. (August 1978) BALL, R.D. Morse theory with applications and extensions. 